\title{
The Effect of Total Hip Arthroplasty on Sports and Work Participation: A Systematic Review and Meta-Analysis
}

\author{
Alexander Hoorntje ${ }^{1,2,3}$ (I) Kim Y. Janssen ${ }^{1} \cdot$ Stefan B. T. Bolder $^{2}$. \\ Koen L. M. Koenraadt ${ }^{2} \cdot$ Joost G. Daams $^{4} \cdot$ Leendert Blankevoort $^{1,3}$. \\ Gino M. M. J. Kerkhoffs ${ }^{1,3}$ - P. Paul F. M. Kuijer ${ }^{5}$
}

Published online: 24 April 2018

(C) The Author(s) 2018

\begin{abstract}
Background Total hip arthroplasty (THA) is a successful procedure to treat end-stage hip osteoarthritis. The procedure is increasingly performed in adults of working age, who often wish to return to sports (RTS) and return to work (RTW). However, a systematic overview of the evidence on RTS and RTW after THA is lacking.

Objectives Our aim was to systematically review (1) the extent to which patients RTS and RTW after THA, including (2) the time to RTS and RTW.
\end{abstract}

Electronic supplementary material The online version of this article (https://doi.org/10.1007/s40279-018-0924-2) contains supplementary material, which is available to authorized users.

Alexander Hoorntje

a.hoorntje@amc.uva.nl

1 Department of Orthopaedic Surgery, Academic Medical Center, Amsterdam Movement Sciences, University of Amsterdam, Meibergdreef 9, 1105 AZ Amsterdam, The Netherlands

2 Department of Orthopaedic Surgery, Foundation FORCE (Foundation for Orthopaedic Research Care and Education), Amphia Hospital, Molengracht 21, 4818 CK Breda, The Netherlands

3 Academic Center for Evidence-Based Sports Medicine (ACES), Meibergdreef 9, 1105 AZ Amsterdam, The Netherlands

4 Medical Library, Academic Medical Center, University of Amsterdam, Meibergdreef 9, 1105 AZ Amsterdam, The Netherlands

5 Coronel Institute of Occupational Health, Amsterdam Public Health Research Institute, Academic Medical Center, University of Amsterdam, Meibergdreef 9, 1105 AZ Amsterdam, The Netherlands
Methods We searched MEDLINE and Embase from inception until October 2017. Two authors screened and extracted the data, including study information, patient demographics, rehabilitation protocols and pre- and postoperative sports and work participation. Methodological quality was assessed using the Newcastle-Ottawa scale. Data on pre- and postoperative sports and work participation were pooled using descriptive statistics.

Results A total of 37 studies were included, of which seven were prospective studies and 30 were retrospective studies. Methodological quality was high in 11 studies, moderate in 16 studies, and low in ten studies. RTS was reported in 14 studies. Mean RTS was $104 \%$ to the pre-surgery level and $82 \%$ to the pre-symptomatic sports level. Time to RTS varied from 16 to 28 weeks. RTW was reported in 23 studies; the mean was $69 \%$. Time to RTW varied from 1 to 17 weeks.

Conclusion A great majority of patients RTS and RTW after THA within a timeframe of 28 and 17 weeks, respectively. For the increasingly younger THA population, this is valuable information that can be used in the preoperative shared decision-making process. 


\section{Key Points}

Eight out of ten patients return to a sports level equal to their pre-symptomatic level after total hip arthroplasty. A return to high-impact sports activities is less likely but is definitely possible in experienced patients.

Overall, seven out of ten patients return to work after total hip arthroplasty. However, modern-day studies showed a mean return to work of $86 \%$. This might be attributed to the increase in total hip arthroplasty in patients aged $<65$ years as well as more liberal work recommendations.

Preoperative sports participation and lower age are predictive of a successful return to sports.

Preoperative sick leave and a high workload are predictive of no return to work.

\section{Introduction}

Total hip arthroplasty (THA) to treat severe osteoarthritis (OA) of the hip joint is one of the most successful orthopedic procedures performed nowadays [1]. The use of THA has skyrocketed in recent decades. In the USA, utilization rates of THA doubled from 102 to 210 per 100,000 between 2000 and 2011 [2, 3]. Likewise, THA utilization rates have been steadily increasing in other developed countries, including the UK, Australia, and most European countries [2]. Numerous reasons for the increasing incidence of THA exist, including the ageing society and the growing prevalence of obesity [4]. Another important factor is patients' participation in an active lifestyle and in highly demanding work and sports activities, both at younger and at older ages [5]. The largest increase in absolute numbers of THA is observed in patients aged $<$ 65 years $[2,6]$, with the greatest percentage increase in the group aged 45-54 years [3].

There is patient demand for improved recovery after hip arthroplasty. Alterations of the technique with tissuesparing approaches may improve early recovery [7]. Improved bearing materials have shown better outcomes with less wear problems, allowing a return to daily activities with full impact [8]. In a younger and more active patient population, a return to daily activities includes return to sports (RTS) and return to work (RTW) [9, 10]. Although of major importance to the patient, scientific data on RTS and RTW issues after THA have been scarce. A systematic review concerning RTW identified seven studies and found that RTW ranged from 25 to $95 \%$ at 1-12 months postoperatively [11]. Timing of RTW ranged from 1.1 to 13.9 weeks. However, the authors did not pool their data for RTW because of the heterogeneity of included studies and found that the overall methodological quality of the included studies was moderate to low [11]. Since the search for the abovementioned review in 2013, newer studies have also focused on RTW after THA $[12,13]$.

No previous study has systematically summarized the available evidence on RTS after THA. Klein et al. [14] evaluated the allowable or recommended sporting activities after THA based on recommendations from 549 orthopedic surgeons. In general, low-impact activities such as swimming, walking, and dancing were allowed, and intermediate-impact sports such as Pilates, ice-skating, and downhill skiing were allowed in individuals experienced in these activities. There was consensus between the surgeons that high-impact sports were not allowed. However, those recommendations were based on expert opinion and not supported by results from clinical studies. Although some research has been performed on RTS after THA, the actual extent of and time to RTS remain largely unknown $[15,16]$. Furthermore, there appears to be a discrepancy between previous RTS recommendations and the actual sports participation that is achieved by modern-day THA patients [17]. Lastly, prognostic factors for RTS after THA have never been systematically reviewed.

A comprehensive evidenced-based review of the literature on the possibility of returning to both sports and work after THA is lacking. Therefore, the purpose of the present analysis was to systematically review the available evidence on the extent to which THA patients RTS and RTW and the timing of this return. In addition, reported prognostic factors for RTS and RTW were reviewed. The results of this study may aid the orthopedic surgeon in providing adequate guidance to future patients about the likelihood of RTS and RTW after THA.

\section{Methods}

\subsection{Search Strategy}

The PRISMA (Preferred Reporting Items for Systematic reviews and Meta-Analyses) guidelines were used for this systematic review [18]. A research protocol was developed before the literature search was commenced. This protocol was published online at the PROSPERO International prospective register of systematic reviews (http://www.crd. york.ac.uk/PROSPERO/; registration number CRD42 016052471). A clinical librarian (JD) developed the search 
strategy in close cooperation with the first author. A systematic search in the MEDLINE and Embase databases was performed from inception until 24 October 2017. A scoping search consisting of citation analysis identified a set of relevant references. From this reference set, search concepts and, subsequently, search terms were derived. Main concepts were (sport OR work-related activities) AND $\{[$ (recovery of function OR surgery) AND longitudinal study design] OR (return to sports \& work)\} AND total hip arthroplasty. Details of the search strategy can be found in the Electronic Supplementary Material (ESM), Appendix S1. The reference lists of selected studies were screened to identify additional studies for inclusion. We also performed a cited reference search in Web of Science to identify more recent studies.

\subsection{Eligibility Criteria and Study Selection}

The results of our MEDLINE and Embase searches were cross-checked and duplicate papers were excluded. The titles and abstracts of the remaining papers were screened by two independent reviewers (AH, KJ) for suitability for inclusion. The Rayyan screening tool for systematic reviews was used to screen titles and abstracts [19]. Discrepancies were resolved by discussion; where there was doubt, the article was included in the full-text screening process. One author $(\mathrm{KJ})$ then selected suitable studies based on the eligibility criteria established in the research protocol. This selection was then reviewed by a second author (AH), and discrepancies were resolved by discussion or by consulting a third reviewer (PK). Inclusion criteria were as follows: observation or intervention studies, describing patients with hip OA who underwent THA, who were participating in sports or working before surgery and intended to RTS and/or RTW after surgery. No restrictions were placed on language or publication year. Review articles were excluded for data extraction, but their references were checked for additional studies that were not identified in our primary search. Exclusion criteria were no primary diagnosis of hip OA, hip resurfacing arthroplasty, and no specific data about RTS or RTW.

\subsection{Outcome Measures}

The primary outcome was the percentage of patients RTS and/or RTW, and the timing of RTS/RTW. The secondary outcomes were specific activity outcome measures, including the University of California Los Angeles (UCLA) activity score (1-10, where $1=$ no physical activity and $10=$ extremely active) [20] and the Grimby scale (1-6, where $1=$ hardly any physical activity and 6 = regular hard exercise) [21]. The Reichsausschuss für
Arbeitszeitermittlung (REFA; German workload classification) classification system was used $(0=$ work with no physical demand and $4=$ work with most heavy physical demand) as a work-related outcome measure.

\subsection{Data Extraction}

Data were systematically extracted from the included studies by one author, and this was independently repeated by a second author. Disagreements were resolved by discussion and, if necessary, by consulting a third reviewer. The authors used a standardized data extraction form that included the following data: (1) study information: author, year, country; (2) study design and follow-up; (3) information about study population: cohort, population size, sex, age, body mass index (BMI), comorbidities; (4) description of rehabilitation protocols used; (5) definition of outcome measures; (6) preoperative activity and definition, e.g., pre-symptomatic or at time of surgery; (7) postoperative activity; (8) RTS and RTW percentages and time to RTS and RTW; (9) confounding factors taken into account in the study, such as sex, age, BMI, motivation, surgeon's advice, preoperative sports participation, workload or sick leave. When information was missing or unclear, we approached the authors for additional information.

\subsection{Quality Assessment}

We used the Newcastle-Ottawa Scale (NOS) to assess the methodological quality of the studies [22] (ESM Appendix S2). One author (KJ) reviewed the quality of all included studies. This was independently repeated by two authors (AH and $\mathrm{PK}$ ), who each reviewed half of the included studies. Discrepancies were resolved by discussion. With the NOS, every study is assessed on eight items, which are subdivided into three groups: the selection of the study groups (four items), the comparability of the groups (one item), and ascertainment of the outcomes of interest for cohort studies (three items). A maximum of one star can be allotted to every item in the selection of the study groups and ascertainment of the outcome groups of interest for cohort studies. A maximum of two stars can be assigned to the comparability of the groups. Thus, a study can receive a maximum of nine stars. In a previous systematic review, Takahashi and Hashizume [23] defined a study as of high quality if it scored seven or more stars. To further improve the distinction between high- and lowquality studies, we defined high-quality studies as those obtaining eight or nine stars for the present systematic review. A study was rated as moderate quality if it obtained six or seven stars. Studies with five stars or fewer were rated as low quality. 


\subsection{Pooling Data}

Patient demographics were analyzed using descriptive statistics. For pooling of RTS and RTW data, we used the methodology previously described by Witjes et al. [24] and Hoorntje et al. [25] for their studies on RTS and RTW after knee arthroplasty and knee osteotomy. Concerning RTS, studies were included if detailed numbers of patients who participated in specific sports pre- and postoperatively were presented. Sports were categorized as low, intermediate, or high impact based on the classification by Vail et al. [26] (ESM Appendix 3). We calculated pooled RTS percentages using descriptive statistics by comparing pooled pre- and postoperative sports participation data. Finally, these results were described as the average number of sports per patient. RTS percentages were analyzed for all included studies and separately for the high-quality studies. RTW data were pooled using descriptive statistics for studies that provided pre- and postoperative numbers of working patients. RTW percentages were analyzed for all included studies and separately for the high-quality studies. RTW was also analyzed separately for studies published before and after 2000 because of the large increase in THA utilization in patients of working age in the last 2 decades [27]. All statistical analyses were performed using SPSS (version 24.0.; IBM Corp, Armonk, NY, USA).

\section{Results}

\subsection{Literature Search}

Figure 1 presents the PRISMA flowchart of our screening process. Our primary search in the MEDLINE and Embase databases yielded 1514 articles. After removing 498 duplicates, 1016 articles remained. After screening of titles and abstracts, we excluded 924 articles. Thus, 92 full-text articles were screened for eligibility. After full-text screening, 37 studies were included. Reasons for exclusion of full-texts were wrong outcome measure $(n=19)$, wrong study design $(n=15)$, no full text available $(n=11)$, wrong population $(n=5)$, same cohort $(n=4)$, and insufficient data $(n=1)$. Searching the references of the included studies provided one additional study [28].

\subsection{Study Characteristics}

Table 1 presents the data extracted for each of the included studies. The studies were published between 1965 and 2016. Eight of the 37 studies included were published before 2000. Three case-control studies, one cross- sectional study, six prospective cohort studies, 24 retrospective cohort studies, two non-randomized controlled studies, and one randomized prospective study were included. Most studies were written in English $(n=34)$, one was written in French, and two were written in German. One study was performed in each of Australia, Denmark, Finland, Greece, Italy, and Switzerland; two were performed in each of Canada, France, Japan, Poland, Spain, Sweden, and the Netherlands; five each were performed in Germany and the UK; and seven were performed in the USA. Of these 37 studies, 13 reported RTS [29-41], 22 reported RTW [12, 13, 42-61], and two reported both RTS and RTW [28, 42].

The total number of included patients was 6485, with 3066 males (47\%) and 3016 females (47\%). Five studies did not report the sex distribution in their cohort, thus sex was unknown for $6 \%$ of the included patients $[30,31,36,38,40]$. The mean duration of follow-up was 3.8 years (range $0.25-11$ ), with one study not reporting time to follow-up [56]. The mean age across studies ranged from 38 to 71 years, with a total age range of 14-98 years. Patients' BMI was specified in 14 studies, with mean BMI varying from 22 to $32 \mathrm{~kg} / \mathrm{m}^{2}$. One study provided information on comorbidities [33]. The authors described that, of the included 420 patients, $7 \%$ had diabetes, $45 \%$ had hypertension, and $11 \%$ had gout. The approach was described in ten studies: one study used a direct anterior approach, two used an anterolateral approach, three used a posterolateral approach, three used a posterior approach, and one used a two-incision approach. The rehabilitation protocol was described in 13 studies. Immediate full weight bearing was allowed in seven studies [29, 32, 35, 43, 51-53] and partial weight bearing was recommended for 3-6 weeks in four studies [37, 40, 46, 54]. Two studies compared restricted and unrestricted movement protocols $[49,52]$. The use of crutches was advised for 4-8 weeks in three studies $[32,35,40]$.

\subsection{Methodological Quality}

Table 2 summarizes the results of the quality assessment. The methodological quality was rated as high in 11 studies, moderate in 16 studies, and low in ten studies. The lowest risk of bias was found for the item "selection of the cohort" (36 studies scored a star), the item "ascertainment of exposure" (34 studies scored a star), and the item "demonstration that the outcome of interest (RTS/RTW) was not present at the start of the study" (36 studies scored a star). The highest risk of bias was found for the item "assessment of outcome," for which ten studies scored a star (Table 2). 

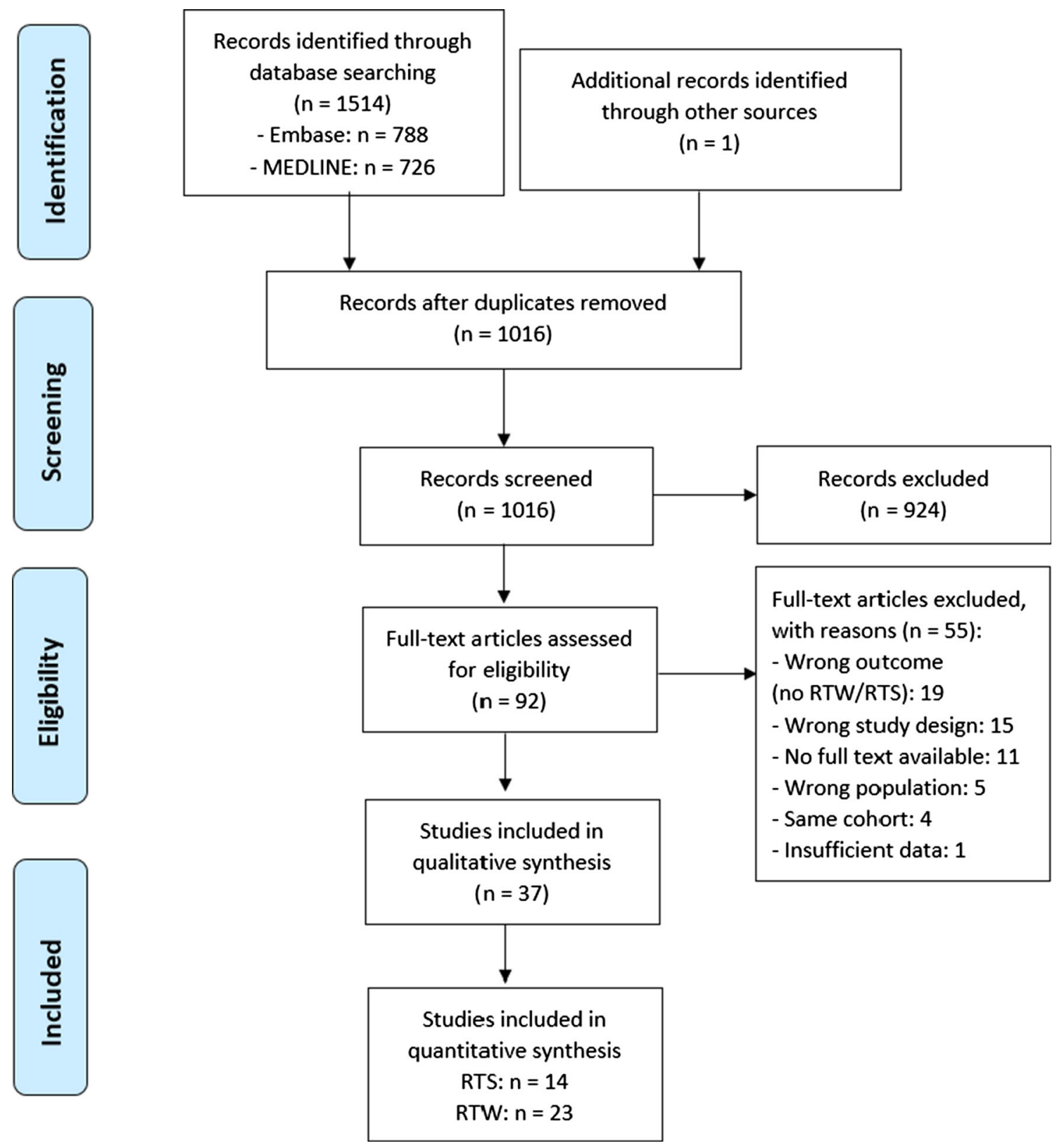

Fig. 1 PRISMA (Preferred Reporting Items for Systematic reviews and Meta-Analyses) flow diagram. $R T S$ return to sports, $R T W$ return to work

\subsection{Return to Sports}

Of 15 studies that reported RTS and time to RTS, 14 reported the percentage of patients that RTS. Mean RTS percentages varied from 43 to $>144 \%$, the latter indicating that more patients participated in sports activities postoperatively than preoperatively (Table 1). Ten studies describing the preoperative sports level as the moment before surgery (pre-surgery level) reported RTS percentages from 48 to $>100 \%$. Four studies describing preoperative sports participation as the moment before the onset of restricting hip symptoms (pre-symptomatic level) reported that 43, 82, 86, and $100 \%$ could RTS
[30, 34, 40, 41]. For the two high-quality studies, Huch et al. [33] and Schmidutz et al. [37], RTS was $>100 \%$ and $98 \%$, respectively, relative to the pre-surgery level. In addition, Huch et al. [33] reported RTS with lifetime sports participation as a reference level and found an average RTS of $53 \%$. Five studies reported time to RTS, with the average being 21.0 weeks (range 15.5-28.0) [30, 35, 36, $39,42]$. No studies with a low risk of bias reported time to RTS. In addition, Chatterji et al. [31], Innmann et al. [34], and Schmidutz et al. [37] reported the cumulative percentage of patients that had returned at different time points (Table 1). 


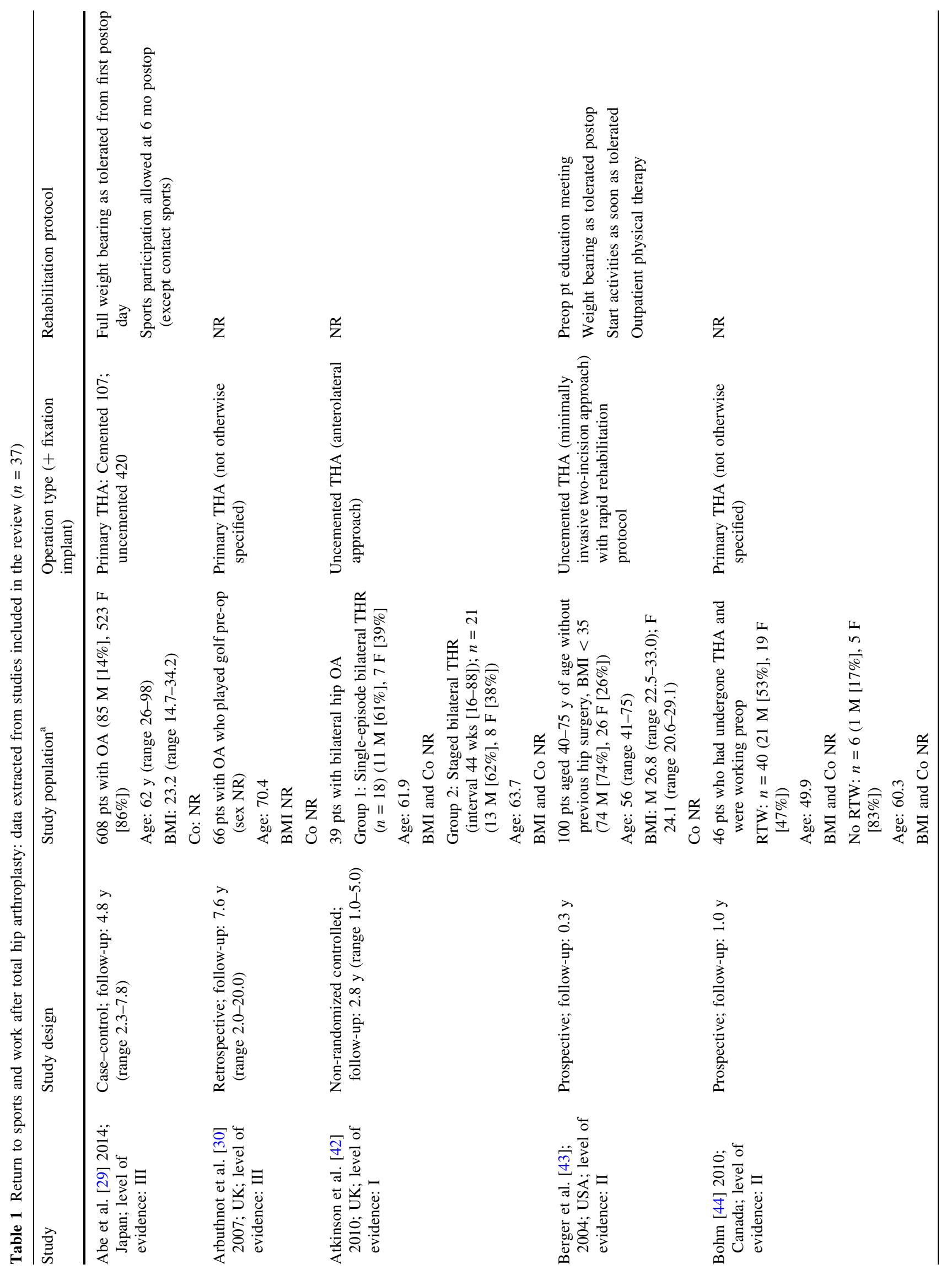




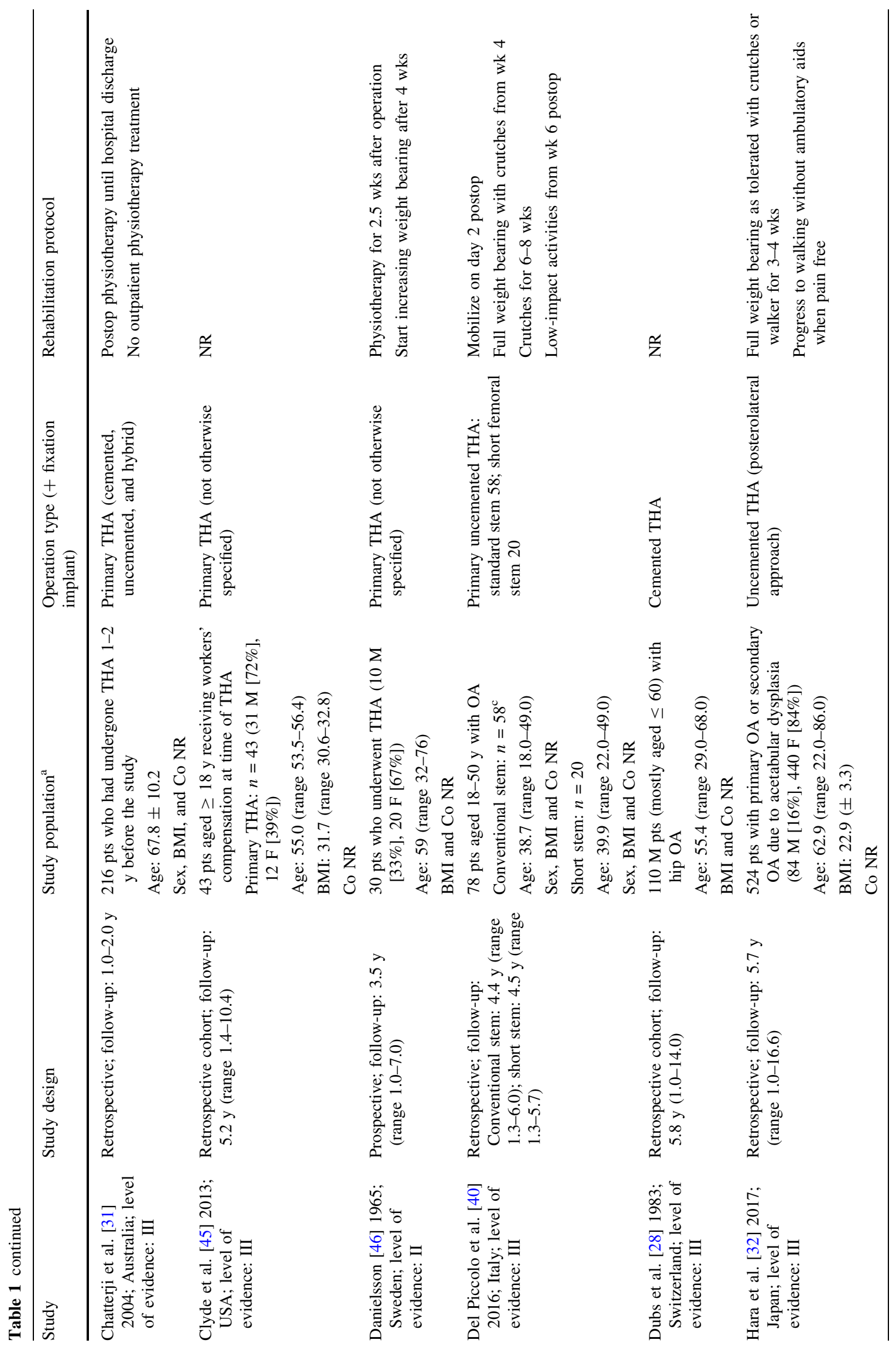




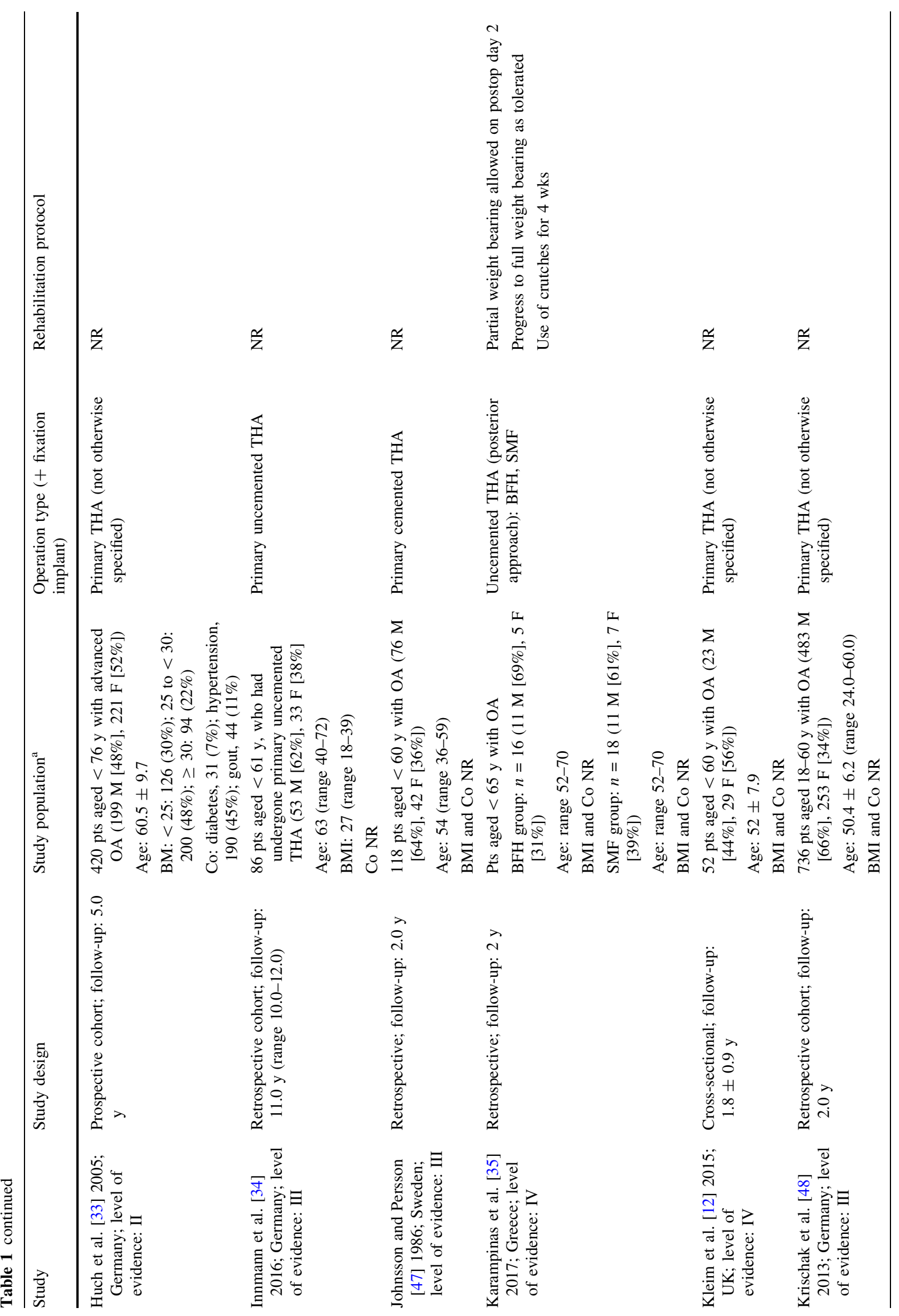




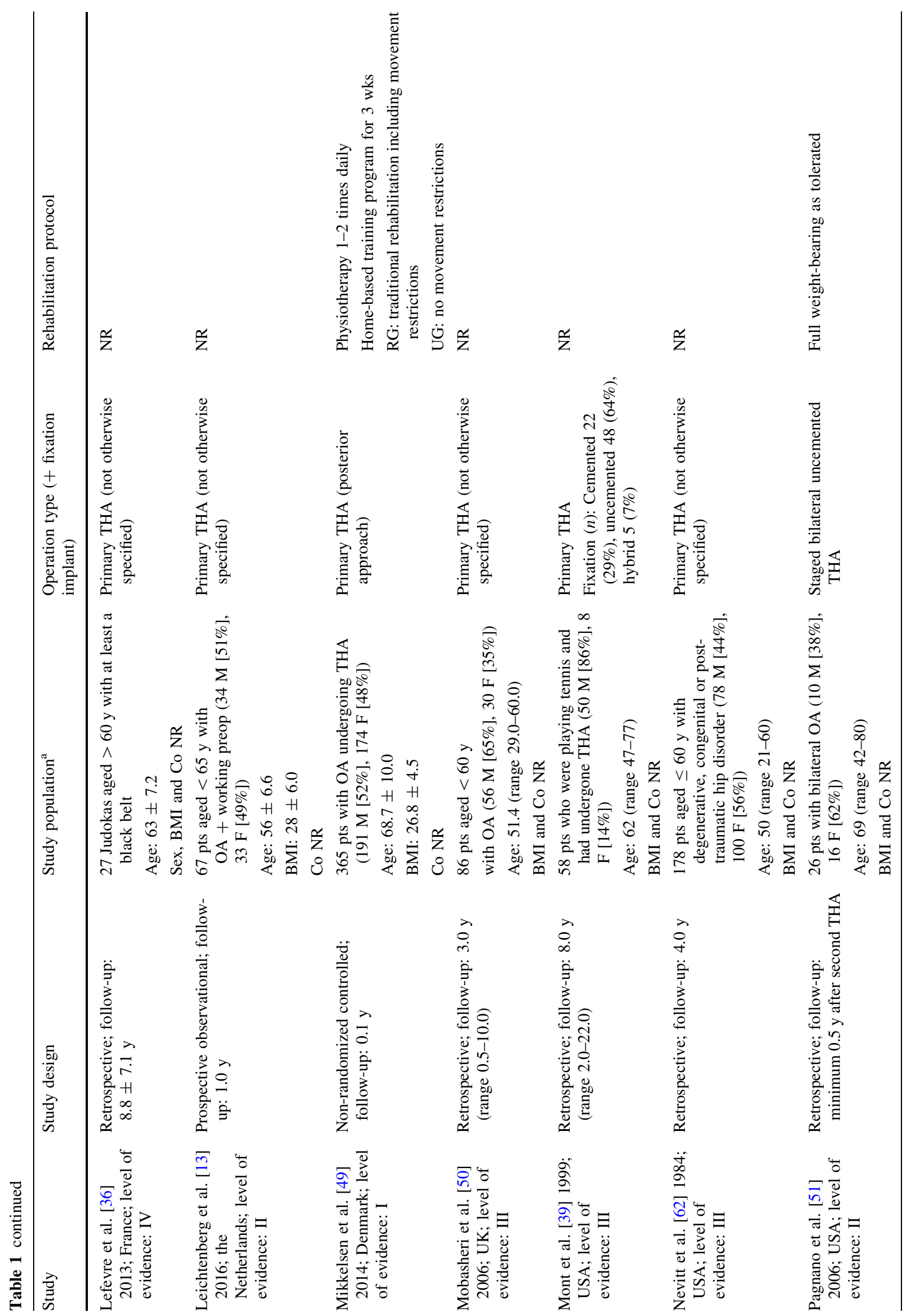




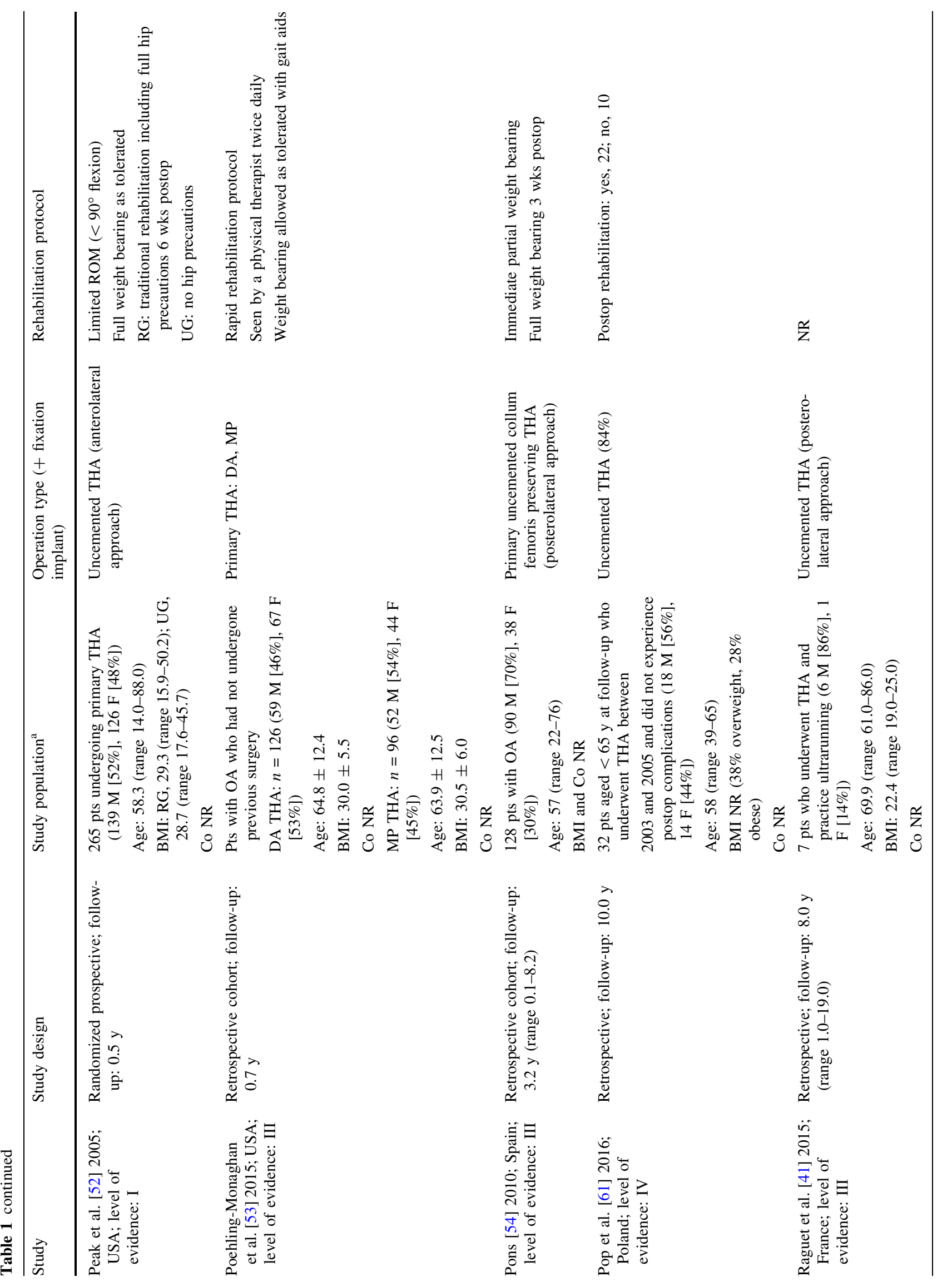




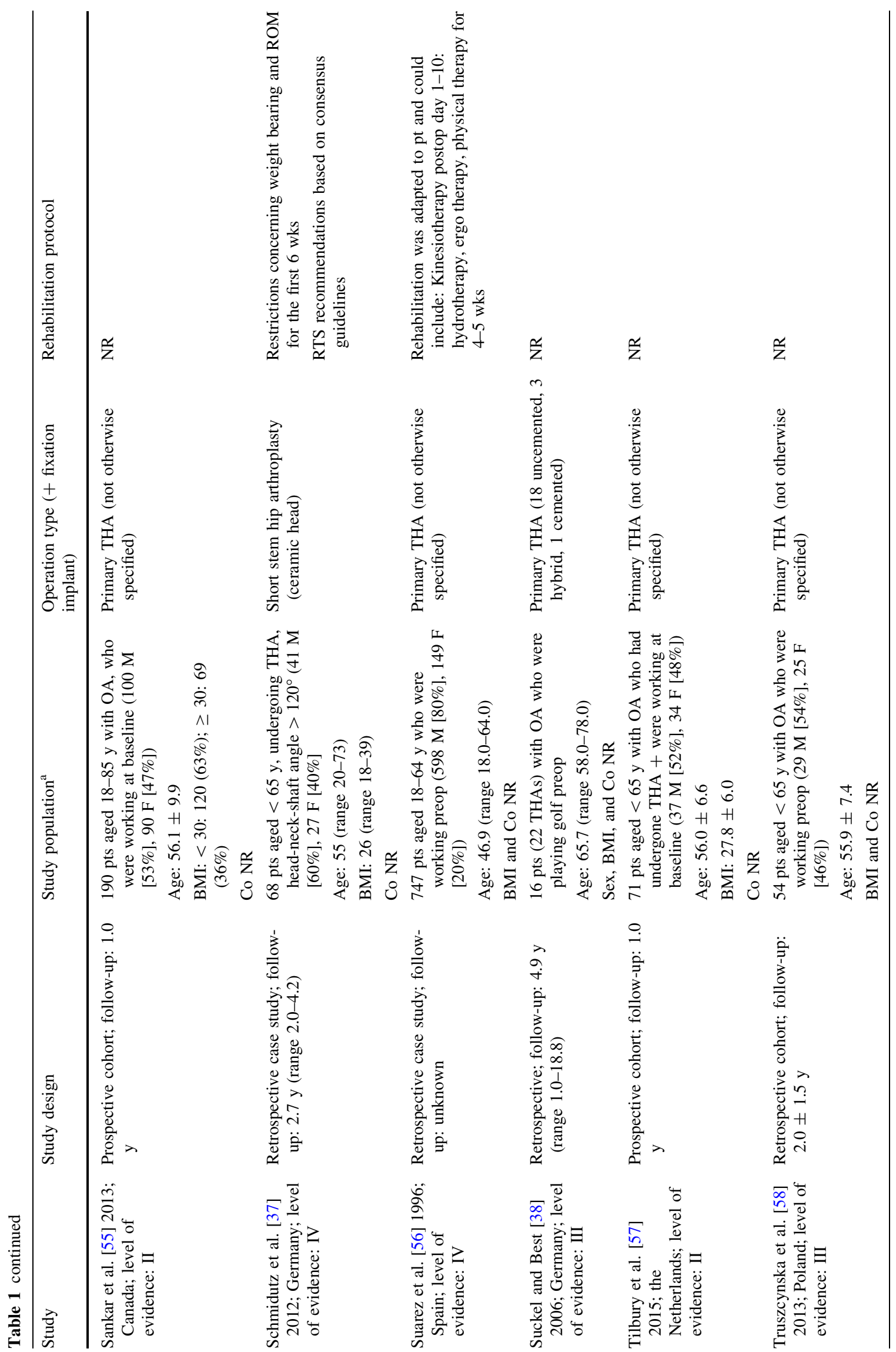




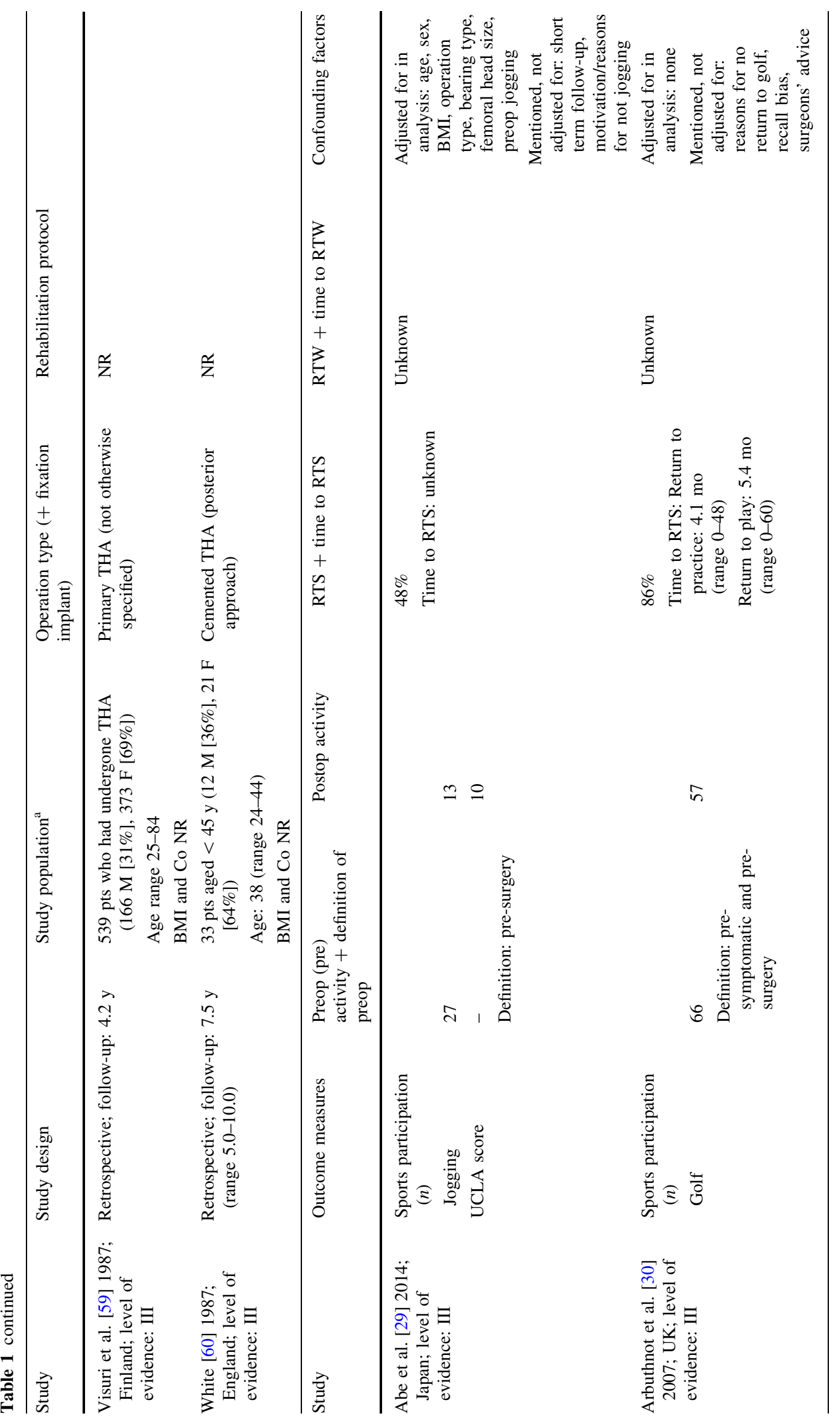




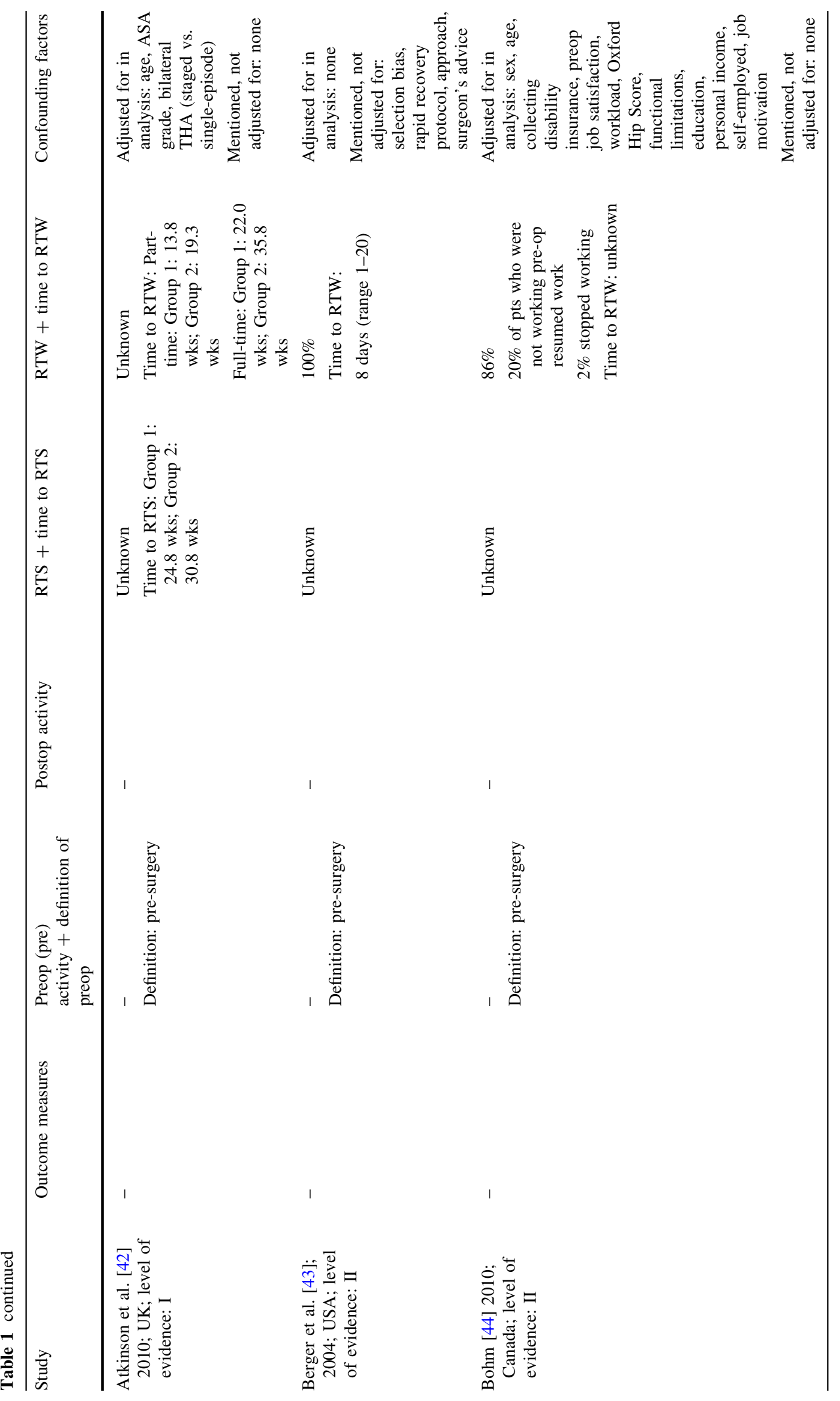




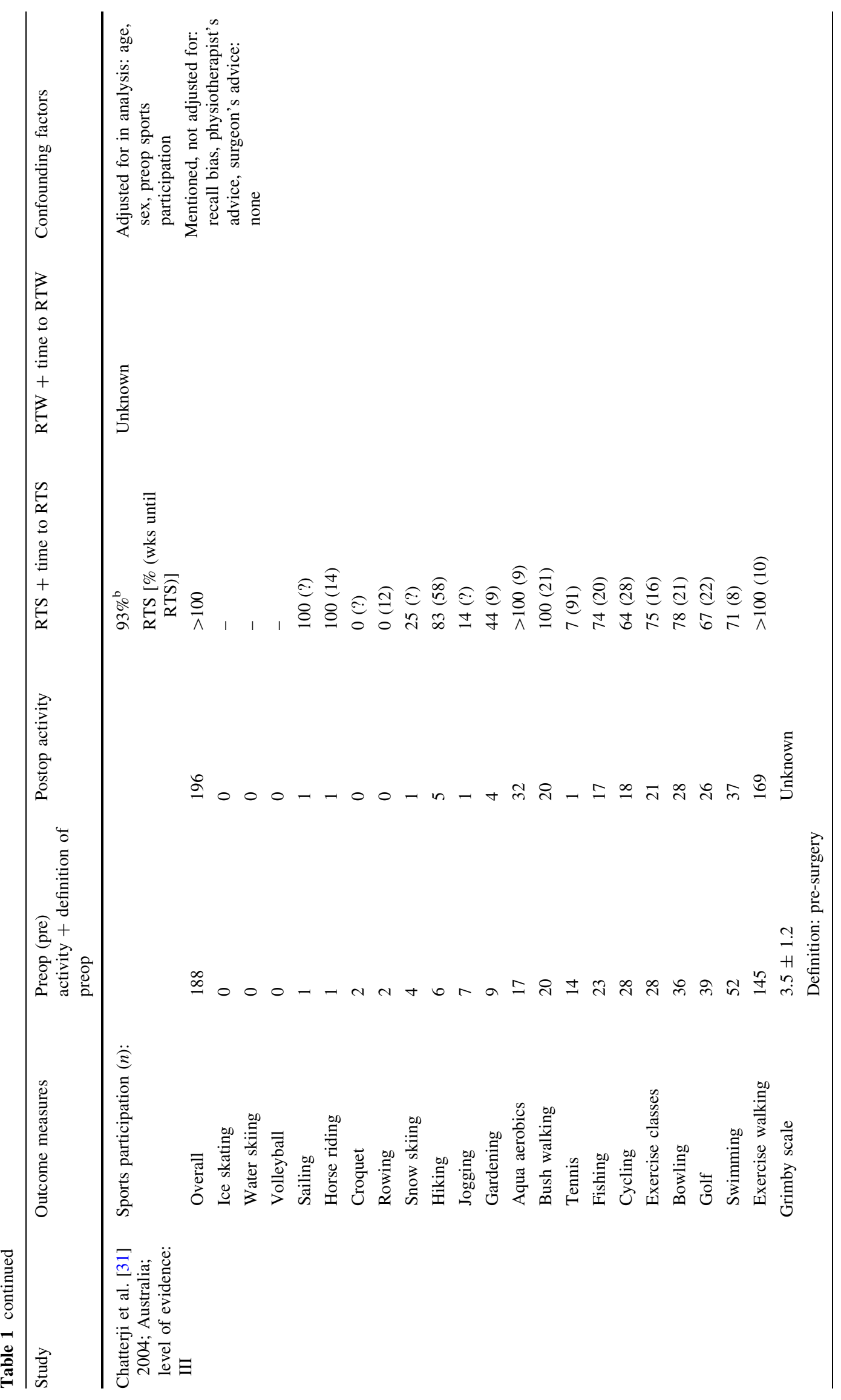




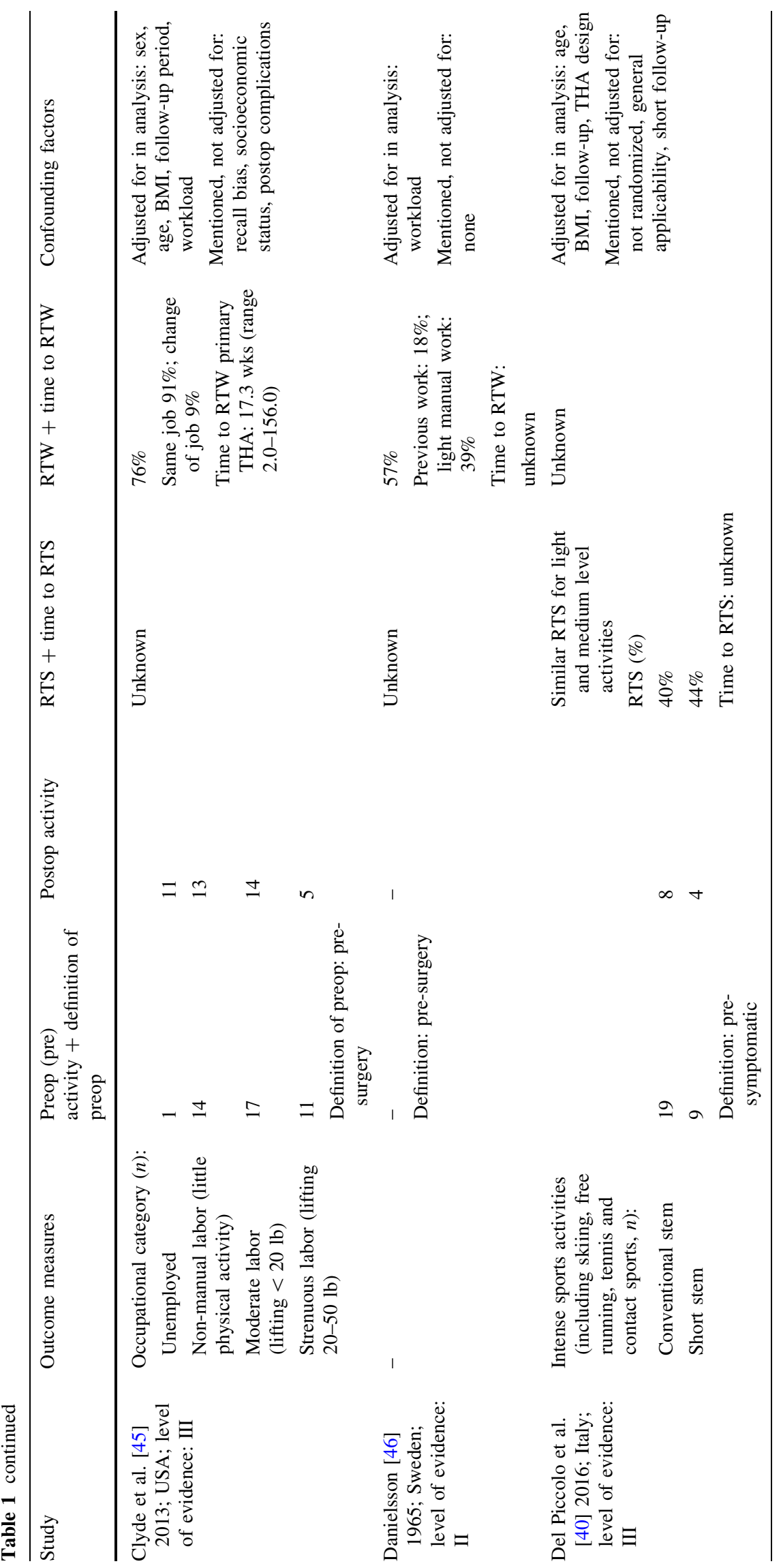




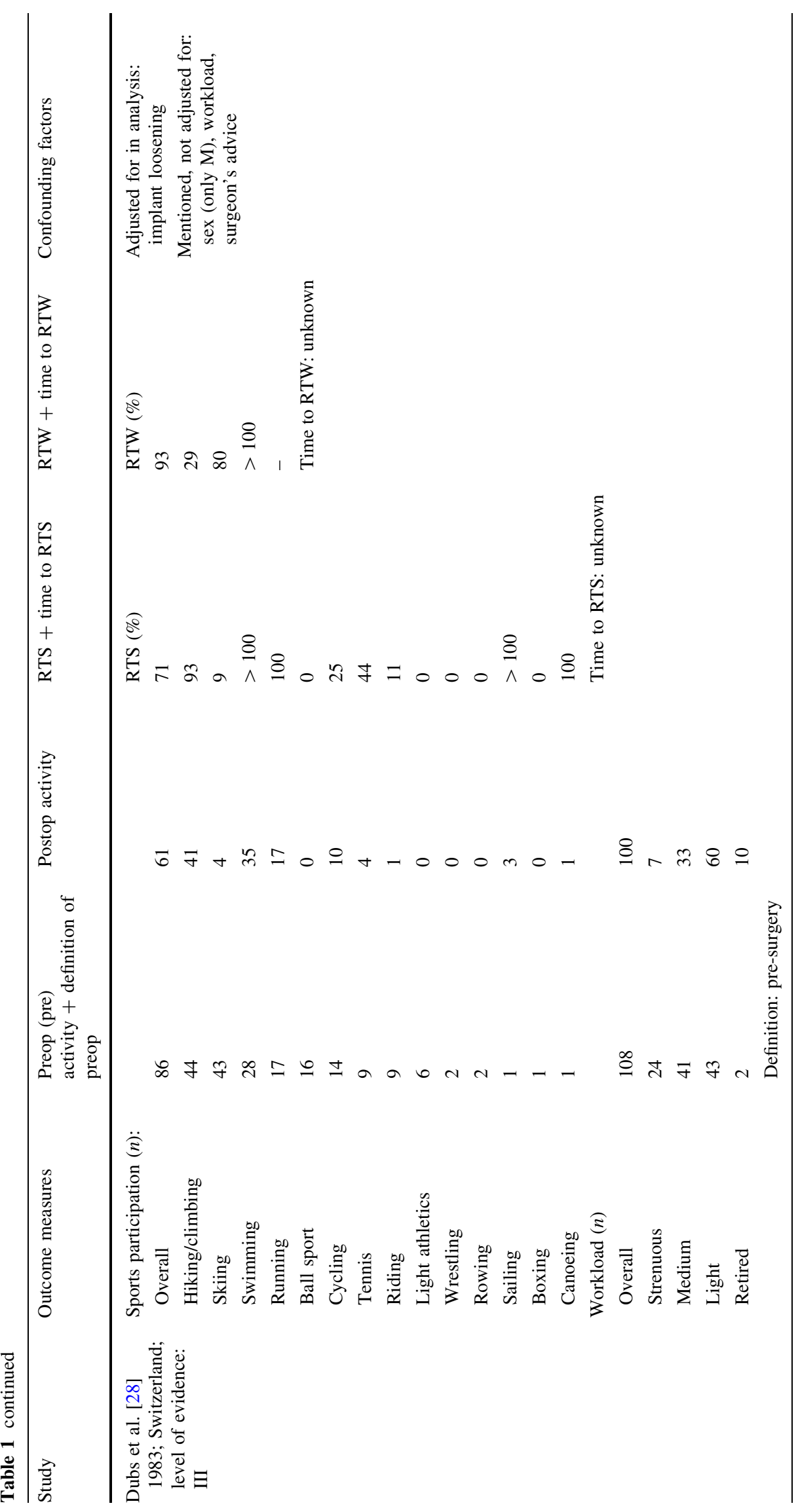




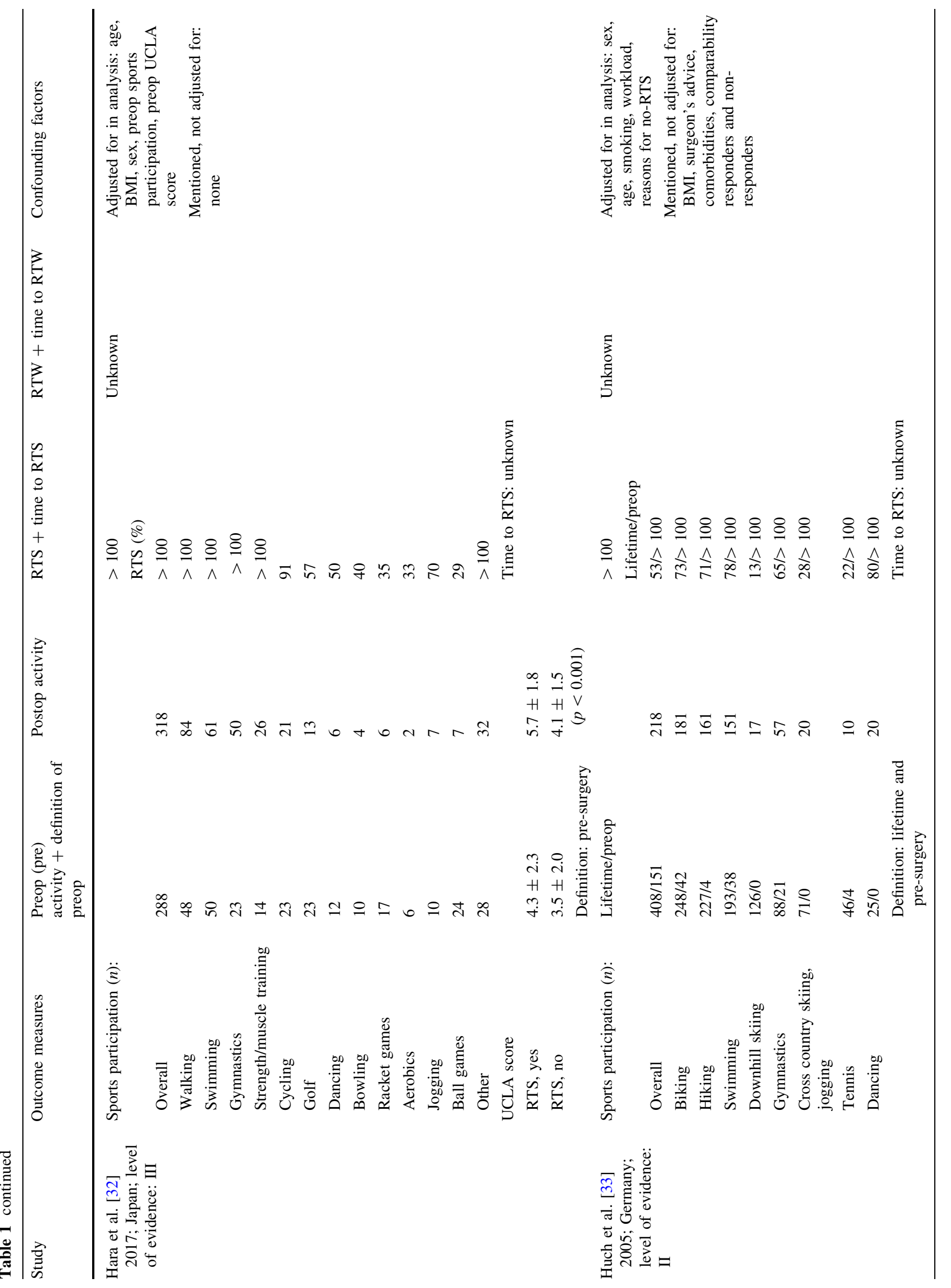




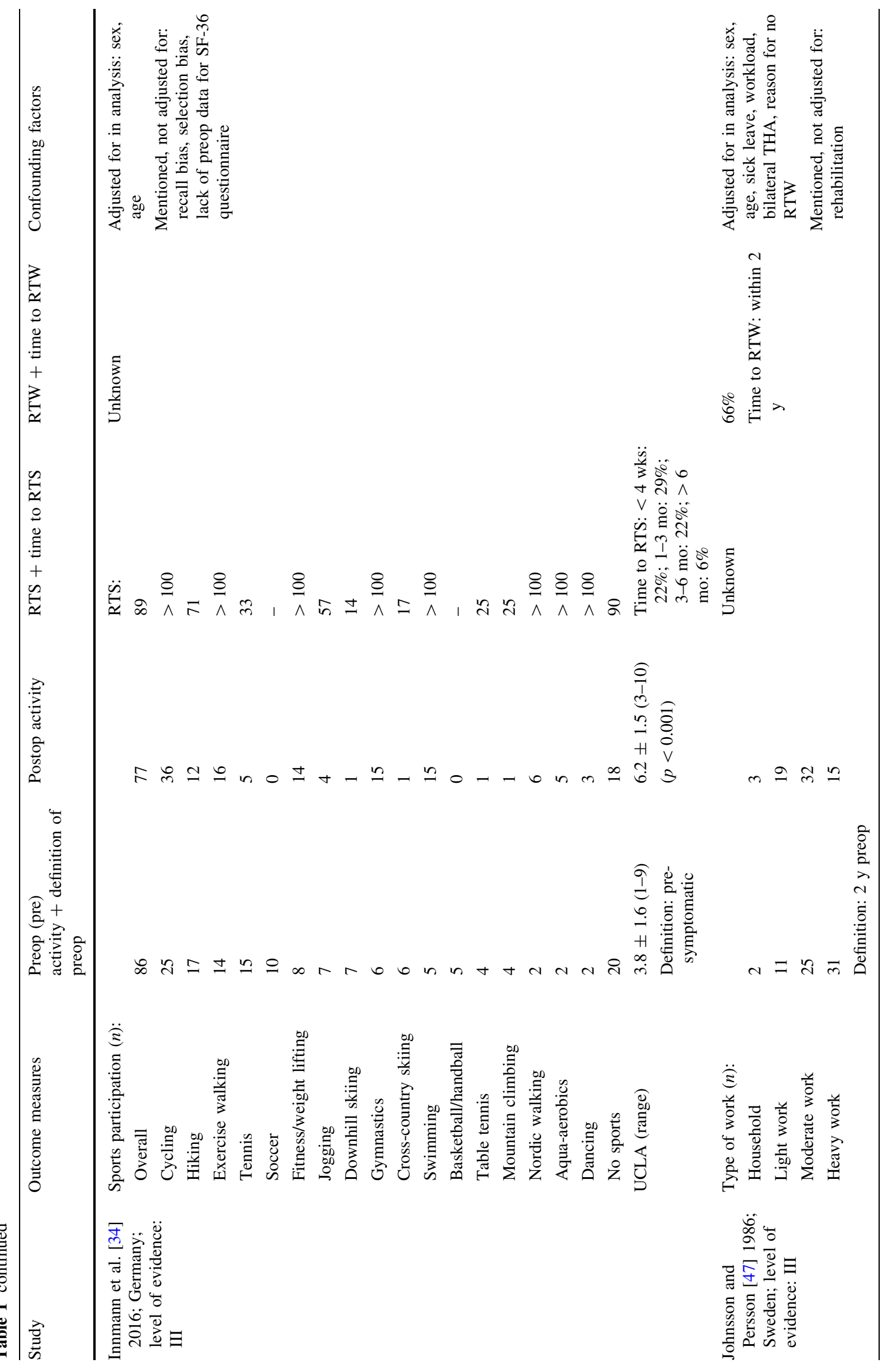




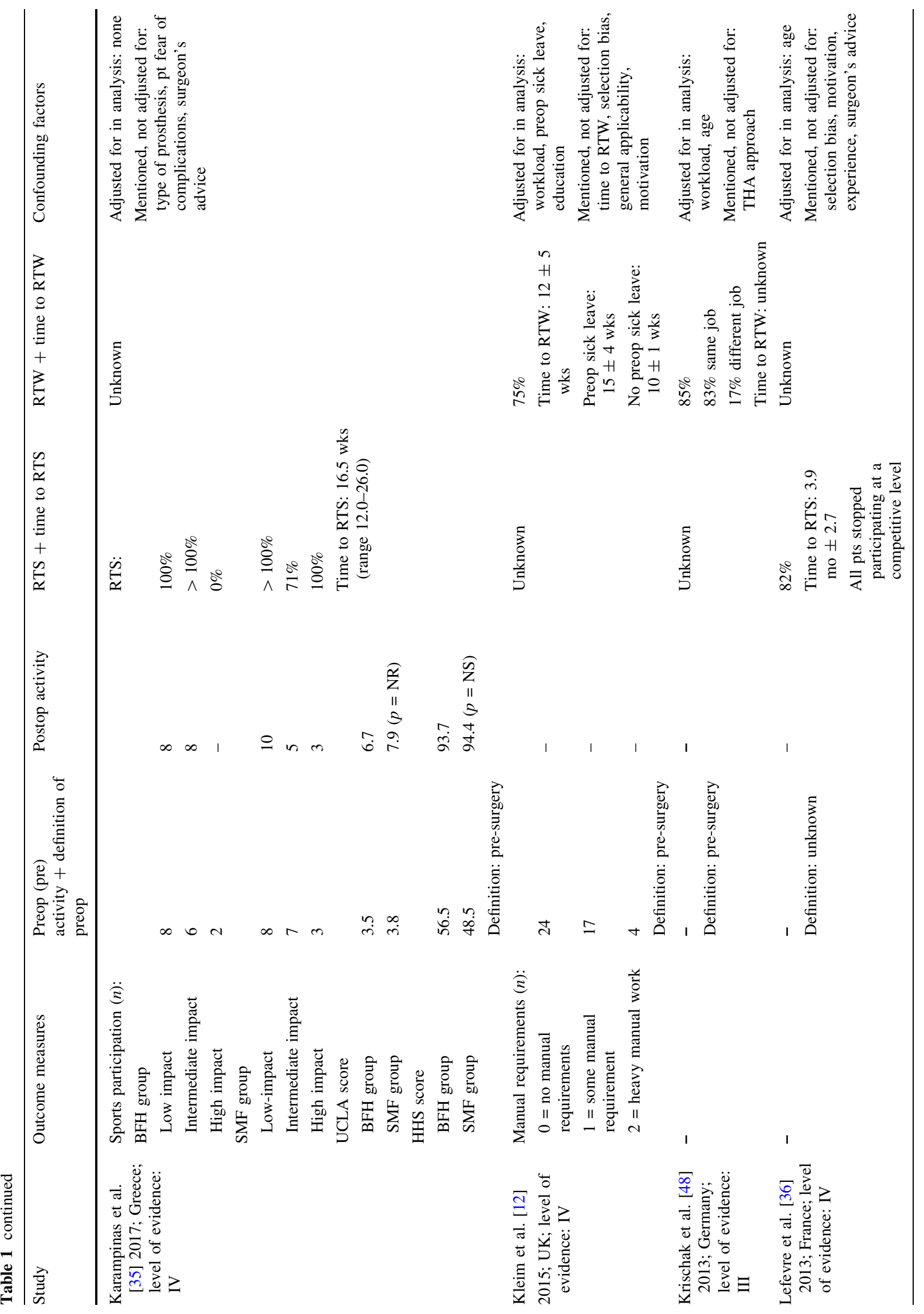




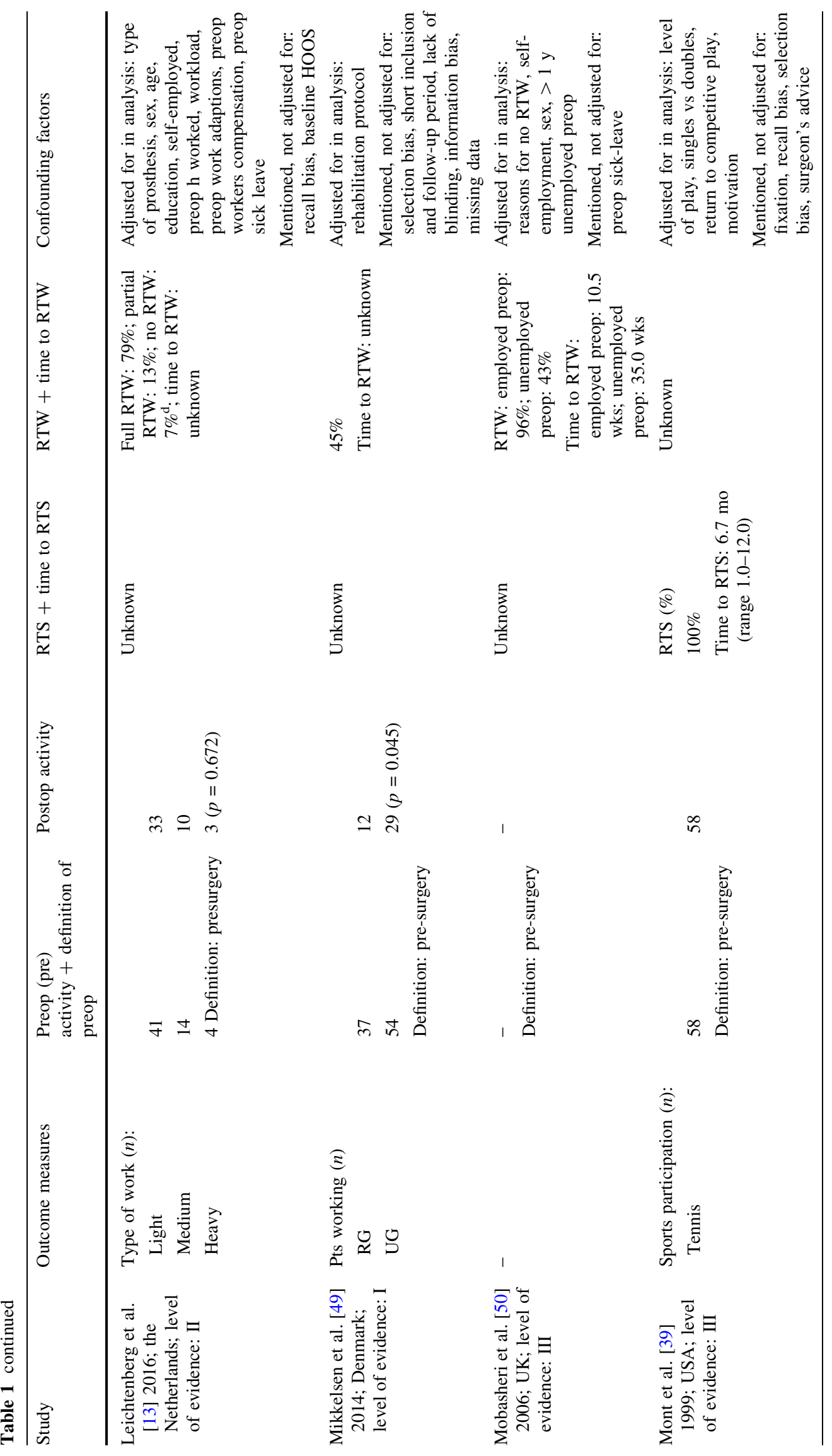




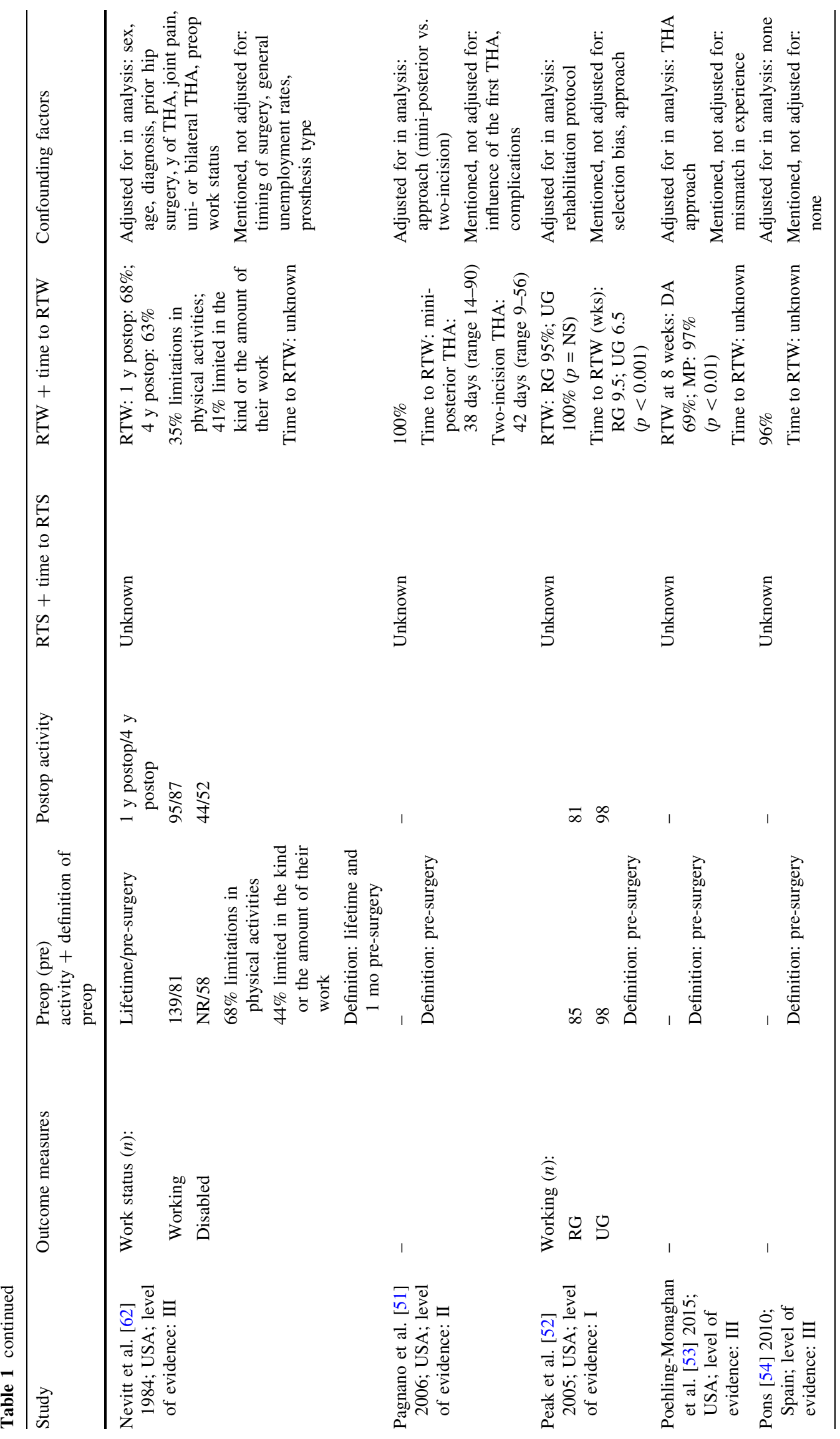




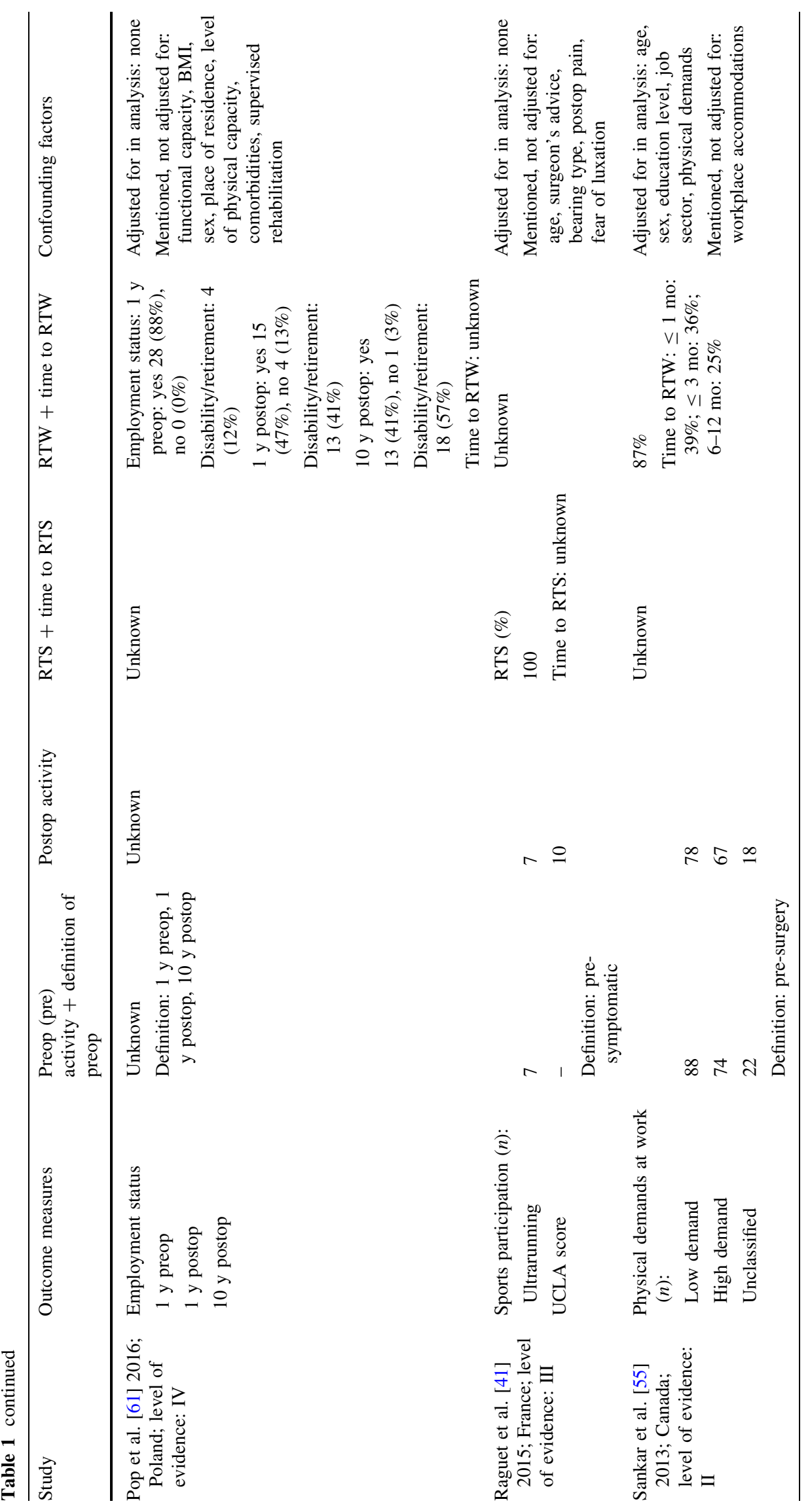




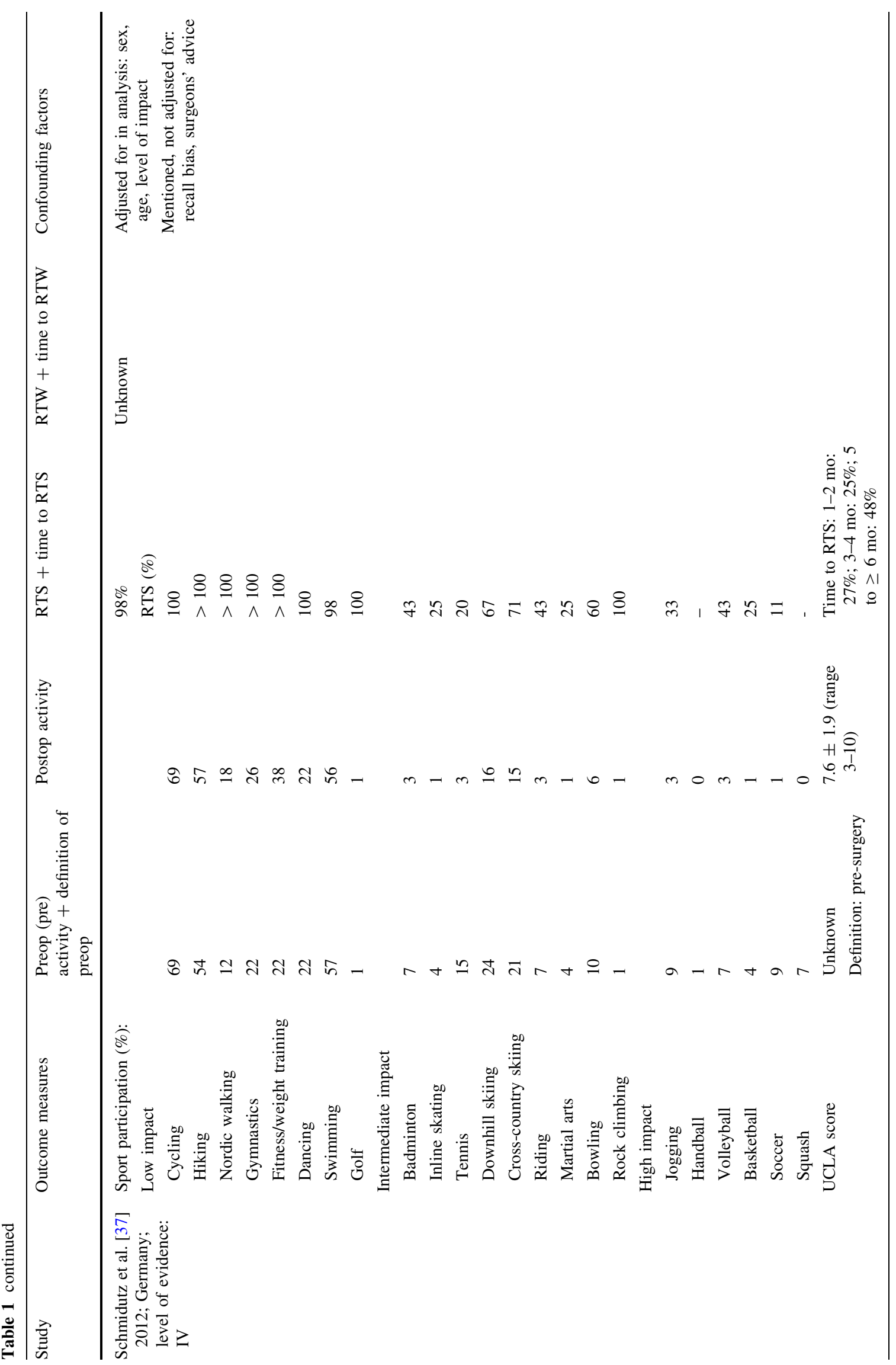




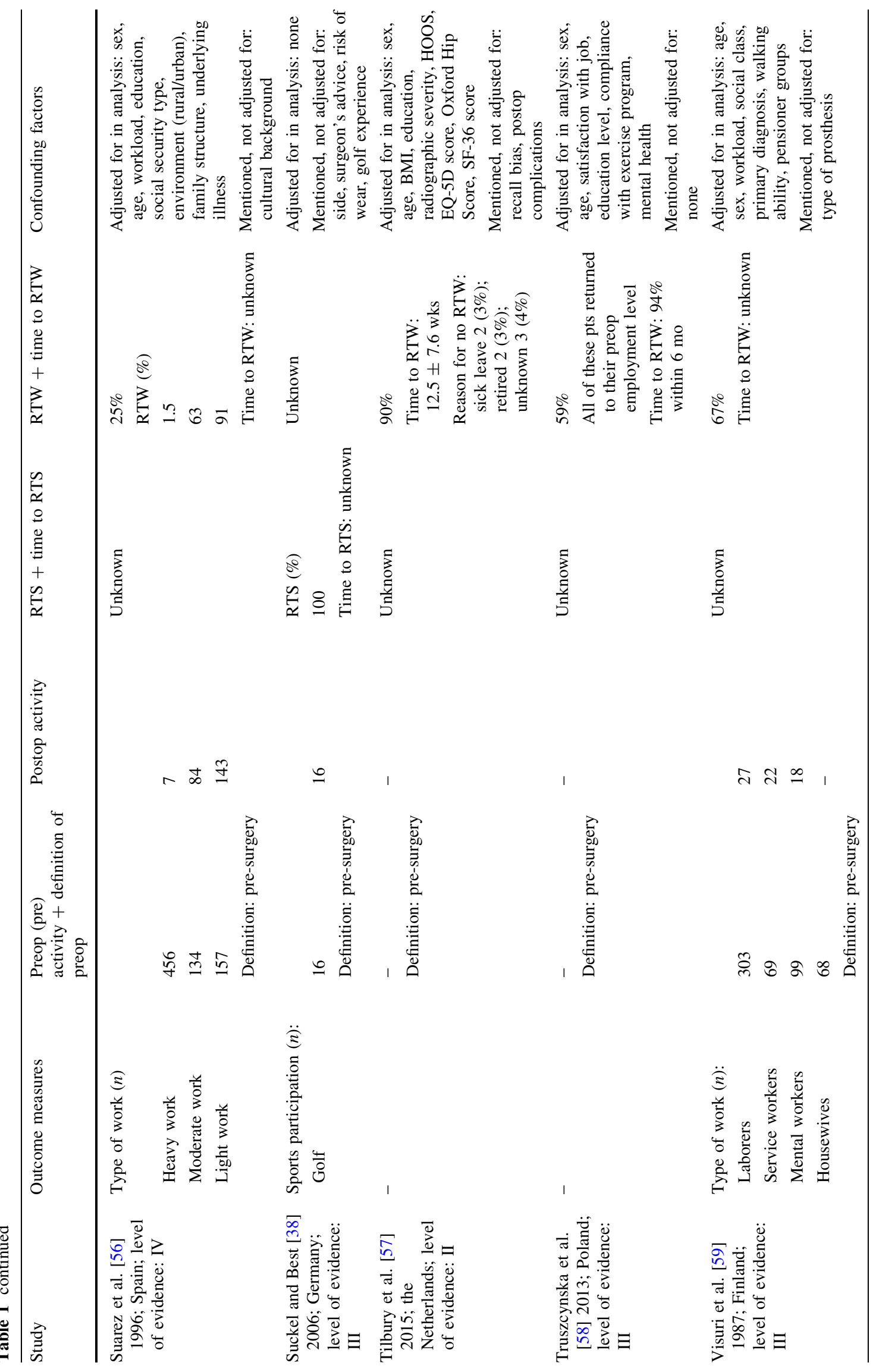




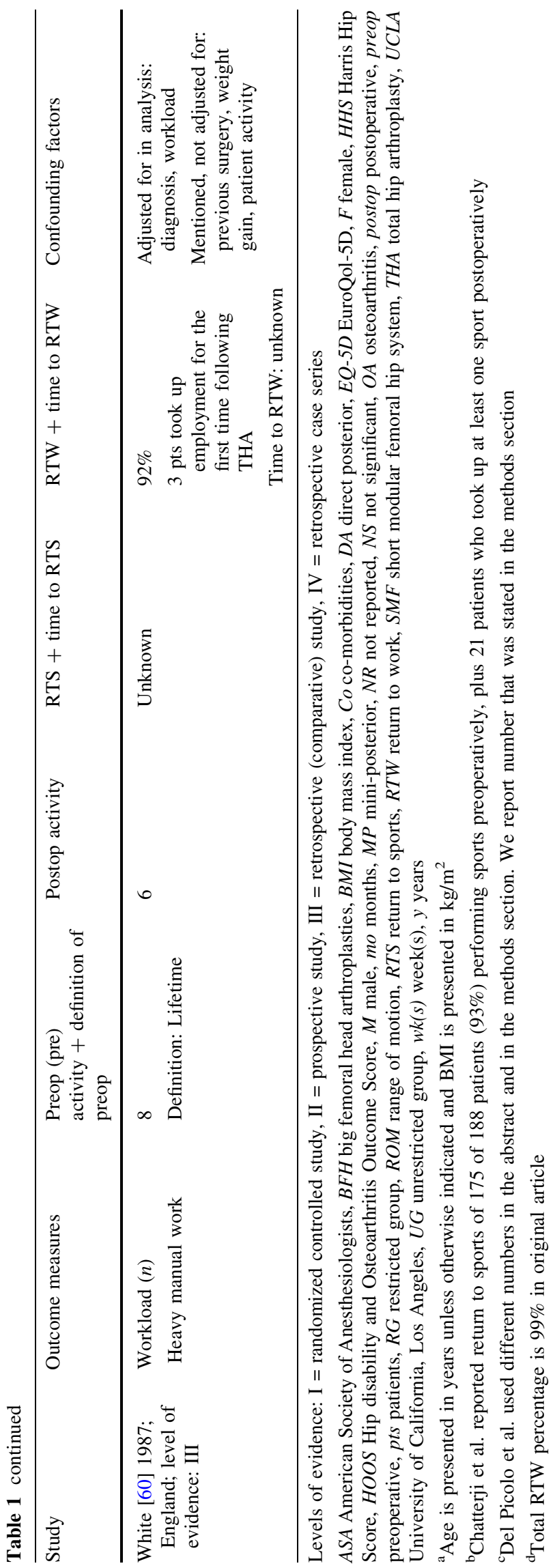

Data could be pooled for 14 studies that reported exact numbers of patients participating in sports pre- and postoperatively (Table 3 ), including 2318 patients (60\% male, median age 62.9 years). Overall, RTS was $100 \%$, but this percentage depended on the definition of the preoperative sports level. Average return to pre-surgery sports level was $104 \%$, and average return to pre-symptomatic sports level was $82 \%$. Both high-quality studies reported the return to pre-surgery sports level and found an average RTS of $131 \%$ (Table 3 ). In addition, one high-quality study also reported return to lifetime sports level, with an average RTS of $53 \%$ (Table 3).

In total, 11 studies described specific numbers of sports that were practiced pre- and postoperatively (Table 4), including 1605 patients $(65 \%$ male, median age 63.0 years). Preoperatively, 1605 patients practiced an average of 1.1 sports, including $62 \%$ low-impact sports, $24 \%$ intermediate-impact sports, and 14\% high-impact sports. Postoperatively, 1605 patients practiced an average of 1.0 sports, including $69 \%$ low-impact sports, $23 \%$ intermediate-impact sports, and $8 \%$ high-impact sports (Table 4). In the high-quality studies, 488 patients practiced an average of 1.3 sports preoperatively, including $52 \%$ low-impact sports, 37\% intermediate-impact sports, and $11 \%$ high-impact sports. Postoperatively, 488 patients practiced an average of 1.1 sports, including $57 \%$ lowimpact sports, $40 \%$ intermediate-impact sports, and $3 \%$ high-impact sports.

\subsection{Return to Work}

Of 24 studies that reported RTW and time to RTW, 23 reported the mean percentage of RTW, which varied from 25 to $>122 \%$, the latter indicating that more patients worked postoperatively than preoperatively (Table 1). For the high-quality studies, mean RTW percentages were 66, $67,68,85,87,87,90,93$, and $122 \%$, respectively [13, 44, 47, 48, 50, 55, 57, 59, 62]. Time to RTW was reported in eight studies, and the mean duration of inability to work varied from 1 to 17 weeks. Pooling of studies, including 3536 patients $(53 \%$ male, median age 52.7 years), showed that 3097 patients worked preoperatively and overall RTW was 69\% (Table 5). For the highquality studies, a mean RTW of $83 \%$ was found. A large difference in RTW was found between studies published before or after 2000 , with a mean RTW of $44 \%$ for studies published in or before 2000 and $86 \%$ for those published after 2000 (Table 5). Pooling of studies that reported time to RTW showed a mean inability to work of 8.9 weeks. For the high-quality studies $(n=2)$, a mean inability to work of 11.4 weeks was found [50,57]. 
Table 2 Methodological assessment according to the Newcastle-Ottawa scale

\begin{tabular}{|c|c|c|c|c|c|c|c|c|c|}
\hline \multirow[t]{2}{*}{ Study } & \multicolumn{4}{|l|}{ Selection } & \multirow[t]{2}{*}{ Comparability } & \multicolumn{3}{|l|}{ Outcome } & \multirow{2}{*}{$\begin{array}{l}\text { Total } \\
\text { score }^{\mathrm{a}}\end{array}$} \\
\hline & Representativeness & Selection & Ascertainment & $\begin{array}{l}\text { Outcome of } \\
\text { interest }\end{array}$ & & Assessment & FU & $\begin{array}{l}\text { Adequacy } \\
\text { of FU }\end{array}$ & \\
\hline Abe et al. [29] & $*$ & $*$ & $*$ & $*$ & $* *$ & - & $*$ & - & 7 \\
\hline $\begin{array}{l}\text { Arbuthnot et al. } \\
\text { [30] }\end{array}$ & $*$ & $*$ & $*$ & $*$ & $*$ & - & $*$ & $*$ & 7 \\
\hline $\begin{array}{l}\text { Atkinson et al. } \\
\text { [42] }\end{array}$ & $*$ & $*$ & $*$ & $*$ & $*$ & - & $*$ & $*$ & 7 \\
\hline Berger et al. [43] & $*$ & $*$ & $*$ & $*$ & - & $*$ & - & $*$ & 6 \\
\hline Bohm [44] & $*$ & $*$ & $*$ & $*$ & $* *$ & - & $*$ & $*$ & 8 \\
\hline Chatterji et al. [31] & $*$ & $*$ & $*$ & $*$ & $* *$ & - & $*$ & - & 7 \\
\hline Clyde et al. [45] & - & $*$ & $*$ & $*$ & $* *$ & - & $*$ & - & 6 \\
\hline Danielsson [46] & $*$ & $*$ & $*$ & $*$ & $*$ & - & $*$ & $*$ & 7 \\
\hline $\begin{array}{l}\text { Del Piccolo et al. } \\
\text { [40] }\end{array}$ & $*$ & $*$ & $*$ & $*$ & $*$ & $*$ & $*$ & - & 7 \\
\hline Dubs et al. [28] & - & $*$ & $*$ & $*$ & - & - & $*$ & $*$ & 5 \\
\hline Hara et al. [32] & $*$ & $*$ & $*$ & $*$ & $* *$ & - & $*$ & - & 7 \\
\hline Huch et al. [33] & $*$ & $*$ & $*$ & $*$ & $* *$ & - & $*$ & $*$ & 8 \\
\hline Innmann et al. [34] & $*$ & $*$ & $*$ & $*$ & $*$ & - & $*$ & $*$ & 7 \\
\hline $\begin{array}{l}\text { Johnsson and } \\
\text { Persson [47] }\end{array}$ & $*$ & $*$ & $*$ & $*$ & $* *$ & $*$ & $*$ & $*$ & 9 \\
\hline $\begin{array}{l}\text { Karampinas et al. } \\
\text { [35] }\end{array}$ & - & $*$ & $*$ & $*$ & $*$ & - & $*$ & - & 5 \\
\hline Kleim et al. [12] & $*$ & $*$ & $*$ & $*$ & $* *$ & - & - & - & 6 \\
\hline Kirschak et al. [48] & $*$ & $*$ & $*$ & $*$ & $* *$ & $*$ & $*$ & $*$ & 9 \\
\hline Lefevre et al. [36] & - & $*$ & - & $*$ & $*$ & - & $*$ & - & 4 \\
\hline $\begin{array}{l}\text { Leichtenberg et al. } \\
\text { [13] }\end{array}$ & $*$ & $*$ & $*$ & $*$ & $* *$ & - & $*$ & $*$ & 8 \\
\hline $\begin{array}{l}\text { Mikkelsen et al. } \\
\text { [49] }\end{array}$ & - & $*$ & $*$ & $*$ & $*$ & - & - & $*$ & 5 \\
\hline $\begin{array}{l}\text { Mobasheri et al. } \\
{[50]}\end{array}$ & $*$ & $*$ & $*$ & $*$ & $* *$ & - & $*$ & $*$ & 8 \\
\hline Mont et al. [39] & - & $*$ & - & $*$ & $*$ & - & $*$ & - & 4 \\
\hline Nevitt et al. [62] & $*$ & $*$ & $*$ & $*$ & $* *$ & - & $*$ & $*$ & 8 \\
\hline Pagnano et al. [51] & - & $*$ & $*$ & $*$ & $*$ & - & - & $*$ & 5 \\
\hline Peak et al. [52] & $*$ & $*$ & $*$ & $*$ & - & $*$ & - & - & 5 \\
\hline $\begin{array}{l}\text { Poehling- } \\
\text { Monaghan et al. } \\
\text { [53] }\end{array}$ & $*$ & - & $*$ & - & - & - & - & - & 2 \\
\hline Pons [54] & $*$ & $*$ & $*$ & $*$ & - & $*$ & $*$ & - & 6 \\
\hline Pop et al. [61] & - & $*$ & $*$ & $*$ & $*$ & - & $*$ & - & 5 \\
\hline Raguet et al. [41] & - & $*$ & $*$ & $*$ & $*$ & - & $*$ & $*$ & 6 \\
\hline Sankar et al. [55] & $*$ & $*$ & $*$ & $*$ & $* *$ & - & $*$ & $*$ & 8 \\
\hline $\begin{array}{l}\text { Schmidutz et al. } \\
\text { [37] }\end{array}$ & $*$ & $*$ & $*$ & $*$ & $* *$ & - & $*$ & $*$ & 8 \\
\hline Suarez et al. [56] & $*$ & $*$ & $*$ & $*$ & $* *$ & $*$ & - & - & 7 \\
\hline Suckel et al. [38] & - & $*$ & - & $*$ & - & - & $*$ & - & 3 \\
\hline Tilbury et al. [57] & $*$ & $*$ & $*$ & $*$ & $* *$ & - & $*$ & $*$ & 8 \\
\hline $\begin{array}{l}\text { Truszczynska et al. } \\
\text { [58] }\end{array}$ & $*$ & $*$ & $*$ & $*$ & $*$ & $*$ & $*$ & - & 7 \\
\hline Visuri et al. [59] & $*$ & $*$ & * & $*$ & $* *$ & * & $*$ & $*$ & 9 \\
\hline
\end{tabular}


Table 2 continued

\begin{tabular}{|c|c|c|c|c|c|c|c|c|c|}
\hline \multirow[t]{2}{*}{ Study } & \multicolumn{4}{|l|}{ Selection } & \multirow[t]{2}{*}{ Comparability } & \multicolumn{3}{|l|}{ Outcome } & \multirow{2}{*}{$\begin{array}{l}\text { Total } \\
\text { score }^{\mathrm{a}}\end{array}$} \\
\hline & Representativeness & Selection & Ascertainment & $\begin{array}{l}\text { Outcome of } \\
\text { interest }\end{array}$ & & Assessment & FU & $\begin{array}{l}\text { Adequacy } \\
\text { of FU }\end{array}$ & \\
\hline White [60] & - & $*$ & * & * & $*$ & $*$ & $*$ & $*$ & 7 \\
\hline
\end{tabular}

$F U$ follow-up

- indicates no stars

${ }^{a}$ We considered a study to be of high quality when the total score was eight or nine stars, moderate quality when the total score was six or seven stars, and low quality when the total score was five stars or fewer

Table 3 Pooled data for number of patients participating in any sport pre- and postoperatively

\begin{tabular}{|c|c|c|c|}
\hline \multirow[t]{2}{*}{ Preoperative reference for RTS } & \multicolumn{2}{|c|}{ No. of pts participating in any sport } & \multirow[t]{2}{*}{ RTS $(\%)$} \\
\hline & Preoperatively & Postoperatively & \\
\hline Overall (14 studies) & 1125 & 1130 & 100 \\
\hline Pre-surgery participation as reference for RTS (10 studies) & 938 & 977 & 104 \\
\hline Pre-symptomatic participation as reference for RTS (4 studies) & 187 & 153 & 82 \\
\hline High-quality studies; pre-surgery participation as reference for RTS (2 studies) & 214 & 280 & 131 \\
\hline High-quality studies; lifetime participation as reference for RTS ${ }^{\mathrm{a}}$ (1 study) & 408 & 218 & 53 \\
\hline
\end{tabular}

No. number, $p t(s)$ patient(s), RTS return to sports

${ }^{a}$ Huch et al. [33] reported both the pre-surgery and lifetime sports participation

Table 4 Pooled data for pre- and postoperative sports participation for different types of sport impact levels

\begin{tabular}{lccccccc}
\hline Impact level & \multicolumn{2}{c}{ Preoperative sports participation (11 studies) } & & \multicolumn{3}{c}{ Postoperative sports participation (11 studies) } \\
\cline { 2 - 3 } & Sports $(n)$ & Pts $(n)$ & Average sports/pt, $n(\%)$ & & Sports $(n)$ & Pts $(n)$ & Average sports/pt, $n(\%)$ \\
\hline Low (e.g., cycling, swimming, golfing) & 1115 & 1605 & $0.69(62)$ & 1090 & 1605 & $0.68(69)$ \\
Intermediate (e.g., hiking, downhill skiing) & 427 & 1605 & $0.27(24)$ & 372 & 1605 & $0.23(23)$ \\
High (e.g., tennis, running, ball sports) & 250 & 1605 & $0.16(14)$ & 122 & 1605 & $0.08(8)$ \\
Total & 1792 & 1605 & 1.12 & 1584 & 1605 & 0.99 \\
\hline
\end{tabular}

$p t(s)$ patient(s)

Table 5 Pooled data for return to work and average duration of inability to work

\begin{tabular}{|c|c|c|c|c|c|c|}
\hline & \multicolumn{3}{|c|}{ Number of working patients } & & \multicolumn{2}{|c|}{ Time to RTW } \\
\hline & $\begin{array}{l}\text { Preoperative } \\
(n)\end{array}$ & $\begin{array}{l}\text { Postoperative } \\
(n)\end{array}$ & $\begin{array}{l}\text { RTW } \\
(\%)\end{array}$ & & $\begin{array}{l}\text { Patients } \\
(n)\end{array}$ & $\begin{array}{l}\text { Inability to work } \\
\text { (weeks) }\end{array}$ \\
\hline Overall (23 studies) & 3097 & 2138 & 69 & Overall (8 studies) & 746 & 8.9 \\
\hline High quality (9 studies) & 1492 & 1242 & 83 & High quality (2 studies) & 157 & 11.4 \\
\hline $\begin{array}{l}\text { Published in or before } 2000 \text { (16 } \\
\text { studies) }\end{array}$ & 1238 & 548 & 44 & $\begin{array}{l}\text { Published in or before } 2000(0 \\
\text { studies) }\end{array}$ & - & - \\
\hline Published after 2000 (7 studies) & 1859 & 1590 & 86 & Published after 2000 (8 studies) & 746 & 8.9 \\
\hline
\end{tabular}

$R T W$ return to work

\subsection{Secondary Outcome Measures of Physical Activity}

The UCLA activity score was reported in six studies. Abe et al. [29] compared postoperative joggers with non- joggers and found a significantly higher postoperative UCLA score (mean \pm standard deviation [SD]) of $10 \pm 0$ for joggers $(n=23)$ compared with $6.6 \pm 2.4$ for nonjoggers $(n=585)$. Hara et al. [32] found that both preoperative and postoperative UCLA scores were significantly 
higher in patients who participated in sports postoperatively than in those who did not: $4.3 \pm 2.3$ versus $3.5 \pm 2.0$ preoperatively and $5.7 \pm 1.8$ versus $4.1 \pm 1.5$ postoperatively. Innmann et al. [34] found a significant increase in UCLA score 11 years after THA, from $3.8 \pm 1.6$ preoperatively to $6.2 \pm 1.5$ postoperatively. Karampinas et al. [35] reported that UCLA scores significantly improved from 3.5 to 6.7 (SD unknown) in the big femoral head group and from 3.8 to 7.9 (SD unknown) in the short metaphyseal stem group. Raguet et al. [41] found a postoperative UCLA score of 10 for seven patients participating in ultrarunning. Lastly, Schmidutz et al. [37] found a postoperative UCLA score of $7.6 \pm 1.9$ after a mean follow-up of 2.7 years. The Grimby scale was reported in one study. Chatterji et al. [31] found a mean postoperative Grimby scale score of $3.5 \pm 1.2$, at 1-2 years after surgery.

\subsection{Confounding Factors: Return to Sports}

Of 15 studies reporting RTS, ten analyzed the effect of one or more confounding factors on RTS. Age was analyzed in eight studies, of which three found an age-dependent decline in RTS [29, 31, 33] and five found no association [32, 34, 37, 40, 42]. Sex was analyzed in five studies: two found a higher RTS in men than in women [29, 33], and three found no association [31, 34, 37]. BMI was analyzed in three studies, which found no association with RTS [29, 32, 40]. Four studies analyzed preoperative sports participation, and all found higher RTS in patients who had already participated in sports preoperatively [29, 31-33]. Additionally, Hara et al. [32] found that a higher preoperative UCLA score was associated with higher RTS. Lastly, one study analyzed the level of impact and found the chance of returning to intermediate- and high-impact sports activities was lower than that of returning to lowimpact activities [37].

\subsection{Confounding Factors: Return to Work}

Of 24 studies reporting RTW, 22 analyzed the effect of one or more confounding factors on RTW. Age was analyzed in 13 studies, of which four found higher RTW in younger patients [13, 44, 48, 59] and nine found no association [36, 42, 45, 47, 55-58, 62]. Sex was analyzed in 12 studies, of which two found that men returned to work faster than did women [50, 55] and ten found no association [13, 44, 45, 47, 56-59, 61, 62]. The three studies that analyzed BMI found no association with RTW [45, 57, 61]. Workload was analyzed in 11 studies, of which six found that higher preoperative workload resulted in lower RTW $[45,47,48,56,59,60]$ and two found that higher workload resulted in longer postoperative inability to work $[12,55]$.
Three studies found no association between workload and RTW [13, 44, 46]. Additionally, three studies found that patients with higher education returned to work faster and more often $[12,13,55]$, and one study found that patients with elementary school education returned to work considerably less often [56]. Three studies found no association between education level and RTW [44, 57, 58]. Five studies analyzed preoperative sick leave and found it to be associated with lower RTW [12, 13, 47, 50, 62]. Additionally, one study found that patients who collected a disability insurance preoperatively were less likely to RTW [44]. Self-employment was analyzed in three studies, of which one found that self-employment resulted in partial or no RTW [13] and two studies found no association $[44,50]$. Job satisfaction was analyzed in two studies, one of which found that $97 \%$ of patients who returned to work were satisfied with their job [58]. In contrast, one study found lower job satisfaction in patients who did RTW [44]. THA approach was analyzed in three studies, one of which found that the mini-posterior approach resulted in higher RTW than did the direct anterior approach [53]. Two other studies found no association [44, 51]. Two studies investigating a restricted compared with an unrestricted rehabilitation protocol found that patients in the unrestricted rehabilitation protocol returned to work more often [49] and faster [52] than those with a restricted protocol.

\section{Discussion}

The aim of the present systematic review and meta-analysis was to investigate the extent of RTS and RTW after THA. Our most important finding was that a large percentage of patients returned to sports and work after THA. Concerning sports participation, the percentage of patients returning to any type of sports activity varied from 43 to $>100 \%$, indicating that more patients participated in sports postoperatively than preoperatively. The average time to RTS was 21 weeks. We also found a decrease in participation in high-impact sports activities and a corresponding increase in participation in low-impact activities. Concerning work resumption, the percentage of patients who could RTW varied from 25 to $>100 \%$, and the average time to RTW was 9 weeks.

\subsection{Return to Sports}

Our meta-analysis revealed an overall RTS percentage of $100 \%$. However, this percentage varied considerably with the preoperative reference point that was used to define sports participation. For instance, 104\% of patients returned to their pre-surgery sports level, whereas only $82 \%$ returned to their pre-symptomatic level. As stated, we 
believe that the pre-symptomatic level represents a more appropriate reference point for RTS, since many patients limit their sports participation before joint replacement because of pain and functional limitations [24, 25]. Still, our finding that more patients participated in sports postoperatively compared with their pre-surgery sports level, with an RTS of $104 \%$, is encouraging. Both high-quality studies used the pre-surgery level and not the pre-symptomatic level as a reference and consequently found very high RTS percentages (98 and 144\%) [33, 37]. Huch et al. [33] also compared lifetime sports participation with postoperative sports participation and found an overall RTS of $53 \%$. However, this is likely an underestimation, since lifetime participation also includes sports that patients practiced in their youth and had stopped practicing for reasons unrelated to their hip. Overall, an RTS of $82 \%$ after THA seems the best estimate, which is in accordance with findings in patients undergoing knee arthroplasty and knee osteotomy [24, 25].

\subsection{Return to Work}

Regarding return to work, our meta-analysis revealed an overall RTW of $69 \%$. The previous systematic review by Tilbury et al. [11] did not pool RTW data, hampering the comparison with the present review. However, the authors described that RTW ranged from 25 to $95 \%$ in seven studies. For the present review, we found 23 studies reporting RTW percentages, which varied from 25 to $>$ $100 \%$. Thus, the newly published studies that we identified reported a range similar to that in the previous review [11]. Given the increasing number of THAs performed in working age patients in the last 2 decades [27], we compared RTW percentages for studies published before and after 2000. As expected, the pooled RTW percentage was considerably higher in studies published after 2000 (86 vs. $44 \%)$. Apart from the increased utilization of THA in patients of working age, this difference might also be attributed to the large increase in less physically demanding jobs in developed countries as well as more liberal recommendations concerning RTW after THA. This was illustrated by findings of two studies describing differences in RTW and time to RTW between a restricted and unrestricted rehabilitation protocol $[49,52]$. Mikkelsen et al. [49] found higher RTW in an unrestricted group (no movement restrictions, RTW of 54\%) than in a restricted group (restricted hip movement for 3 months; RTW of $32 \%$ ). Peak et al. [52] found that patients in an unrestricted group (no movement restrictions) returned to work after 6 weeks compared with 9 weeks in a restricted group (movement restrictions for 6 weeks). Interestingly, none of the other included studies mentioned the effect of rehabilitation protocol or surgeons' advice concerning RTW.
Thus, the effect of different rehabilitation protocols and surgeons' recommendations on RTW and time to RTW is an important topic for future studies. Lastly, one included study reported that 5 of 67 patients $(7 \%)$ did not RTW, whereas nine patients $(13 \%)$ could only partially RTW [13]. Since most patients expressed a preoperative desire to fully RTW, this subtotal loss of work capacity might be clinically relevant. Therefore, future studies should aim to distinguish between a full and partial RTW after hip arthroplasty.

\subsection{Prognostic Factors}

\subsubsection{Prognostic Factors for Return to Sports}

Regarding RTS, increasing age was associated with a decline in RTS in three studies, whereas five studies found no association. However, of those five studies, three limited their inclusion to patients aged $<60$ years $[34,37,40]$ and one study included a small number of patients [42], thus limiting statistical validity. In general, above the age of 65 years, a postoperative decline in sports participation might be expected for THA patients. An important prognostic factor for RTS is preoperative sports participation, which was analyzed by five studies, all of which found higher RTS in patients who had participated in sports preoperatively. This is in line with findings in patients undergoing knee arthroplasty and knee osteotomy [24, 25]. Only one study analyzed the impact level of a sport; the authors found that participation in intermediate- and highimpact sports was associated with lower RTS than participation in low-impact sports.

Remarkably, patients' motivation to RTS, which is a proven prognostic factor for RTS in patients undergoing hip and knee arthroplasty [24, 63], was not mentioned in any of the included studies. Furthermore, surgeons' advice is a main reason for patients to refrain from sports participation after THA [15, 63], but none of the included studies adjusted for this. Compared with the 1999 Hip Society recommendations for athletic activity after THA [64], subsequent expert opinion surveys found an increasing tolerance for and acceptance of granting patients permission to return to higher-impact activities, such as downhill skiing or ice skating [14, 65, 66]. Interestingly, Swanson et al. [65] found that high-volume hip surgeons were more liberal in their recommendations, indicating that, as experience with THA and RTS grows, orthopedic surgeons become less cautious. Thus, a trend of allowing more sports activities after THA can be observed over the past 2 decades. Although our data suggest that patients do engage in intermediate- and high-impact sports activities, we observed a shift from high-impact sports activities to lowimpact sports activities. From a surgeon's point of view, 
this may seem desirable, but it is important to discuss this with the patient before surgery. High-impact sports activities are known to negatively influence THA durability [67]. However, as some of the included studies have shown, a limited proportion of patients do participate in high-impact activities such as tennis, running, and judo. For many of these patients, the possibility of continuing their high-impact activities is the main reason for surgery. It appears that the experience level of patients, which was high in all patients returning to high-impact activities, influences the possibility of RTS. Thus, despite the increased risk of mechanical failure, a return to high-impact activities should not be completely ruled out, especially in experienced patients. The 15 -year survival rate in highly active patients is reported to be approximately $80 \%$ for bearings that were used 20 years ago [68]. The risk of dislocation and fractures is low. To address the needs of active patients, surgeons can use bearings with low wear rates and femoral heads up to $36 \mathrm{~mm}$ in diameter [68]. Future studies are needed to identify the thresholds for physical activity in patients undergoing hip arthroplasty, above which the negative effects in terms of prosthesis wear exceed the positive effects in terms of general health improvement and patient satisfaction $[15,17]$.

\subsubsection{Prognostic Factors for Return to Work}

This systematic review is one of the first studies to systematically summarize the effects of confounding factors on RTW [11,69] and the first to summarize the effects of confounding factors on RTS. These findings provide additional guidance for the orthopedic surgeon and the patient in preoperative counseling and discussing the probability of RTW and RTS. Regarding RTW, a previous literature review identified two studies of confounding factors influencing RTW after THA, both of which found that preoperative sick leave was associated with lower RTW [70]. Another review identified 15 studies describing beneficial or limiting factors for RTW after THA but did not summarize these findings [11]. Our systematic review identified 22 studies reporting on confounding factors for RTW. Preoperative sick leave appears to be a consistent prognostic factor for RTW. All studies that included preoperative sick leave in the analysis found lower RTW for patients who were absent from work preoperatively, which is in line with findings in patients undergoing knee arthroplasty [24, 71, 72]. Furthermore, a higher preoperative workload was associated with lower RTW in most studies. Similarly, a higher level of education, which results in more white-collar jobs and thus a lower physical workload, was associated with higher RTW. Factors such as collecting a preoperative disability pension or disability insurance, job satisfaction, employment versus self- employment, and THA approach, might be associated with RTW. However, only a limited number of studies investigated these factors, and more research is needed to verify this. To this end, future studies of RTW in THA patients should collect and discuss these factors.

\subsection{Limitations}

A limitation of the present analysis is the low availability of high-quality, prospective studies. Most studies had a retrospective design, thus increasing the risk of recall bias. Also, methodological quality was rated as moderate or low in most studies. Furthermore, no validated questionnaires for the assessment of RTS and RTW exist, which hampers comparisons between studies. The development of a core outcome set will allow for more reliable and valid collection of patient data on work and sports participation before and after THA [73].

\section{Conclusion}

Most patients were able to RTS and RTW after THA within a timeframe of 28 and 17 weeks, respectively. Participation in high-impact sports activities is less likely but not impossible. Furthermore, RTW with a high workload or after prolonged preoperative sick leave is less likely. For the increasingly younger THA population, this is valuable information that can be used by the orthopedic surgeon and the patient in the preoperative shared decisionmaking process.

Acknowledgements The authors thank Dr. Abe and Dr. Sakai for kindly providing us with additional information regarding return to sports percentages in their study.

\section{Compliance with Ethical Standards}

Funding No sources of funding were used to assist in the preparation of this article.

Conflict of interest Alexander Hoorntje, Kim Janssen, Stefan Bolder, Koen Koenraadt, Joost Daams, Leendert Blankevoort, Gino Kerkhoffs and Paul Kuijer have no conflicts of interest relevant to the content of this review.

Open Access This article is distributed under the terms of the Creative Commons Attribution 4.0 International License (http:// creativecommons.org/licenses/by/4.0/), which permits unrestricted use, distribution, and reproduction in any medium, provided you give appropriate credit to the original author(s) and the source, provide a link to the Creative Commons license, and indicate if changes were made. 


\section{References}

1. Learmonth ID, Young C, Rorabeck C. The operation of the century: total hip replacement. Lancet. 2007;370:1508-19.

2. Pabinger C, Geissler A. Utilization rates of hip arthroplasty in OECD countries. Osteoarthr Cartil. 2014;22:734-41.

3. Wolford ML, Palso K, Bercovitz A. Hospitalization for total hip replacement among inpatients aged 45 and over: United States, 2000-2010. NCHS Data Brief. United States; 2015. p. 1-8.

4. Pivec R, Johnson AJ, Mears SC, Mont MA. Hip arthroplasty. Lancet. 2012;380:1768-77.

5. Kurtz SM, Lau E, Ong K, Zhao K, Kelly M, Bozic KJ. Future young patient demand for primary and revision joint replacement: national projections from 2010 to 2030. Clin Orthop Relat Res. 2009;467:2606-12.

6. Kurtz S, Ong K, Lau E, Mowat F, Halpern M. Projections of primary and revision hip and knee arthroplasty in the United States from 2005 to 2030. J Bone Jt Surg Am. 2007;89:780-5.

7. Parvizi J, Restrepo C, Maltenfort MG. Total hip arthroplasty performed through direct anterior approach provides superior early outcome: results of a randomized, prospective study. Orthop Clin N Am. 2016;47:497-504.

8. Peters RM, Van Steenbergen LN, Stevens M, Rijk PC, Bulstra SK, Zijlstra WP. The effect of bearing type on the outcome of total hip arthroplasty: analysis of 209,912 primary total hip arthroplasties registered in the Dutch Arthroplasty Register. Acta Orthop. 2017;3674:1-7.

9. Witjes S, van Geenen RC, Koenraadt KL, van der Hart CP, Blankevoort L, Kerkhoffs GMMJ, et al. Expectations of younger patients concerning activities after knee arthroplasty: are we asking the right questions? Qual Life Res. 2017;26:403-17.

10. Wright JG, Rudicel S, Feinstein AR. Ask patients what they want. Evaluation of individual complaints before total hip replacement. J Bone Jt Surg Br. 1994;76:229-34.

11. Tilbury C, Schaasberg W, Plevier JWM, Fiocco M, Nelissen RGHH, Vliet Vlieland TPM. Return to work after total hip and knee arthroplasty: a systematic review. Rheumatology (Oxford). 2014;53:512-25.

12. Kleim BD, Malviya A, Rushton S, Bardgett M, Deehan DJ. Understanding the patient-reported factors determining time taken to return to work after hip and knee arthroplasty. Knee Surg Sports Traumatol Arthrosc. 2015;23:3646-52.

13. Leichtenberg C, Tilbury C, Kuijer P, Verdegaal S, Wolterbeek R, Nelissen R, et al. Determinants of return to work 12 months after total hip and knee arthroplasty. Ann R Coll Surg Engl. 2016;98:387-95.

14. Klein GR, Levine BR, Hozack WJ, Strauss EJ, D'Antonio JA, Macaulay W, et al. Return to athletic activity after total hip arthroplasty. Consensus guidelines based on a survey of the Hip Society and American Association of Hip and Knee Surgeons. J Arthroplasty. 2007;22:171-5.

15. Meira EP, Zeni J. Sports participation following total hip arthroplasty. Int J Sports Phys Ther. 2014;9:839-50.

16. Healy WL, Sharma S, Schwartz B, Iorio R. Athletic activity after total joint arthroplasty. J Bone Jt Surg Am. 2008;90:2245-52.

17. Oehler N, Schmidt T, Niemeier A. Total joint replacement and return to sports. Sportverletzung-Sportschaden. 2016;30:195-203.

18. Moher D, Shamseer L, Clarke M, Ghersi D, Liberati A, Petticrew $\mathrm{M}$, et al. Preferred Reporting Items for Systematic Review and Meta-Analysis Protocols (PRISMA-P) 2015 statement. Syst Rev. 2015;4:1.

19. Ouzzani M, Hammady H, Fedorowicz Z, Elmagarmid A. Rayyan-a web and mobile app for systematic reviews. Syst Rev. 2016;5:210.
20. Zahiri CA, Schmalzried TP, Szuszczewicz ES, Amstutz HC. Assessing activity in joint replacement patients. J Arthroplasty. 1998;13:890-5.

21. Grimby G. Physical activity and muscle training in the elderly. Acta Med Scand Suppl. 1986;711:233-7.

22. Wells GA, Shea B, Connell DO, Peterson J, Welch V, Losos M, et al. The Newcastle-Ottawa Scale (NOS) for assessing the quality of nonrandomised studies in meta-analyses [Internet]. 2012. Available from: http://www.ohrica/programs/clinical_ epidemiology/oxford.asp.

23. Takahashi N, Hashizume M. A systematic review of the influence of occupational organophosphate pesticides exposure on neurological impairment. BMJ Open. 2014;4(6):e004798.

24. Witjes S, Gouttebarge V, Kuijer PPFM, van Geenen RCI, Poolman RW, Kerkhoffs GMMJ. Return to sports and physical activity after total and unicondylar knee arthroplasty: a systematic review and meta-analysis. Sports Med. 2016;46:1-24.

25. Hoorntje A, Witjes S, Kuijer PPFM, Koenraadt KLM, van Geenen RCI, Daams JG, et al. High rates of return to sports activities and work after osteotomies around the knee: a systematic review and meta-analysis. Sports Med. 2017;47:1-26.

26. Vail T, Mallon W, Liebelt R. Athletic activities after joint arthroplasty. Sports Med Arthrosc. 1996;4:298-305.

27. Koenig L, Zhang Q, Austin MS, Demiralp B, Fehring TK, Feng $\mathrm{C}$, et al. Estimating the societal benefits of THA after accounting for work status and productivity: a Markov model approach. Clin Orthop Relat Res. 2016;474:2645-54.

28. Dubs L, Gschwend N, Munzinger U. Sport after total hip arthroplasty. Arch Orthop Trauma Surg. 1983;101:161-9.

29. Abe H, Sakai T, Nishii T, Takao M, Nakamura N, Sugano N. Jogging after total hip arthroplasty. Am J Sports Med. 2014;42:131-7.

30. Arbuthnot JE, McNicholas MJ, Dashti H, Hadden WA. Total hip arthroplasty and the golfer. A study of participation and performance before and after surgery for osteoarthritis. J Arthroplasty. 2007:22:549-52.

31. Chatterji U, Ashworth M, Lewis P, Dobson P. The effect of total hip arthroplasty on recreational and sporting activity. ANZ J Surg. 2004;74(6):446-9.

32. Hara D, Hamai S, Komiyama K, Motomura G, Shiomoto K, Nakashima Y. Sports participation in patients after total hip arthroplasty vs periacetabular osteotomy: a propensity scorematched Asian cohort study. J Arthroplasty. 2018;33(2):423-30.

33. Huch K, Müller KAC, Stürmer T, Brenner H, Puhl W, Günther KP. Sports activities 5 years after total knee or hip arthroplasty: the Ulm Osteoarthritis Study. Ann Rheum Dis. 2005;64:1715-20.

34. Innmann MM, Weiss S, Andreas F, Merle C, Streit MR. Sports and physical activity after cementless total hip arthroplasty with a minimum follow-up of 10 years. Scand J Med Sci Sports. 2016;26:550-6.

35. Karampinas PK, Papadelis EG, Vlamis JA, Basiliadis H, Pneumaticos SG. Comparing return to sport activities after short metaphyseal femoral arthroplasty with resurfacing and big femoral head arthroplasties. Eur J Orthop Surg Traumatol. 2017;27:617-22.

36. Lefevre N, Rousseau D, Bohu Y, Klouche S, Herman S. Return to judo after joint replacement. Knee Surg Sports Traumatol Arthrosc. 2013;21:2889-94.

37. Schmidutz F, Grote S, Pietschmann M, Weber P, Mazoochian F, Fottner A, et al. Sports activity after short-stem hip arthroplasty. Am J Sports Med. 2012;40:425-32.

38. Suckel A, Best R. Der golfsport mit hüft- und knietotalendoprothesen. Sportverletzung-Sportschaden. 2006;20:127-31.

39. Mont MA, Laporte DM, Mullick T, Silberstein CE, Hungerford DS. Tennis after total hip arthroplasty. 1999;27:60-4. 
40. Del Piccolo N, Carubbi C, Mazzotta A, Sabbioni G, Filanti M, Stagni C, et al. Return to sports activity with short stems or standard stems in total hip arthroplasty in patients less than 50 years old. HIP Int. 2016;26(Suppl 1):48-5.

41. Raguet M, Pierson C, Pierson A. Is ultrarunning possible with a total hip arthroplasty? [French]. J Traumatol du Sport. 2015;32:3-14.

42. Atkinson HD, Bailey CA, Willis-Owen CA, Oakeshott RD. Bilateral hip arthroplasty: is 1-week staging the optimum strategy? J Orthop Surg Res. 2010;5:84.

43. Berger RA, Jacobs JJ, Meneghini RM, Della Valle C, Paprosky W, Rosenberg AG. Rapid rehabilitation and recovery with minimally invasive total hip arthroplasty. Clin Orthop Relat Res. 2004(429):239-47.

44. Bohm ER. The effect of total hip arthroplasty on employment. J Arthroplasty. 2010;25:15-8.

45. Clyde CT, Goyal N, Matar WY, Witmer D, Restrepo C, Hozack WJ. Workers' compensation patients after total joint arthroplasty. J Arthroplasty. 2013;28:883-7.

46. Danielsson L. Arthroplasty of the hip according to Thompson and Moore. Acta Orthop Scand. 1965;35:348-57.

47. Johnsson R, Persson BM. Occupation after hip replacement for arthrosis. Acta Orthop. 1986;57:197-200.

48. Krischak G, Kaluscha R, Kraus M, Tepohl L, Nusser M. Rück$\mathrm{kehr}$ in das erwerbsleben nach hüfttotalendoprothese. Unfallchirurg. 2013;116:755-9.

49. Mikkelsen LR, Petersen MK, Mikkelsen S, Mechlenburg I. Does reduced movement restrictions and use of assistive devices affect rehabilitation outcome after total hip replacement? A non-randomized, controlled study. Eur J Phys Rehabil Med. 2014;50:383-93.

50. Mobasheri R, Gidwani S, Rosson J. The effect of total hip replacement on the employment status of patients under the age of 60 years. Ann R Coll Surg Engl. 2006;88:131-3.

51. Pagnano MW, Trousdale RT, Meneghini RM, Hanssen AD. Patients preferred a mini-posterior THA to a contralateral twoincision THA. Clin Orthop Relat Res. 2006;453:156-9.

52. Peak E, Parvizi J, Purtill J, Sharkey P, Hozack WJ, Rothman R. The role of patient restrictions in reducing the preveleance of early dislocation following total hip arthroplasty. J Bone Jt Surg. 2005;87:247-53.

53. Poehling-Monaghan KL, Kamath AF, Taunton MJ, Pagnano MW. Direct anterior versus miniposterior THA with the same advanced perioperative protocols: surprising early clinical results. Clin Orthop Relat Res. 2014;473:623-31.

54. Pons M. Learning curve and short-term results with a short-stem CFP system. HIP Int. 2010;20(Suppl 7):S52-7.

55. Sankar A, Davis AM, Palaganas MP, Beaton DE, Badley EM, Gignac MA. Return to work and workplace activity limitations following total hip or knee replacement. Osteoarthr Cartil. 2013;21:1485-93.

56. Suarez J, Arguelles J, Costales M, Arechaga C, Cabeza F, Vijande M. Factors influencing the return to work of patients after hip replacement and rehabilitation. Arch Phys Med Rehabil. 1996;77:269-72.
57. Tilbury C, Holtslag MJ, Tordoir RL, Leichtenberg CS, Verdegaal SHM, Kroon HM, et al. Outcome of total hip arthroplasty, but not of total knee arthroplasty, is related to the preoperative radiographic severity of osteoarthritis. Acta Orthop. 2016;87:67-71.

58. Truszczyńska A, Rapala K, Tarnowski A, Kucharczyk W. Analysis of factors influencing return to work after total hip arthroplasty. Ortop Traumatol Rehabil. 2013;15:459-67.

59. Visuri T, Koskenvuo M, Lindholm TS. Return to work and working capacity after total hip replacement. Int J Rehabil Res. 1987;10:434-9.

60. White SH. The fate of cemented total hip arthroplasty in young patients. Clin Orthop Relat Res. 1988;(231):29-34.

61. Pop T, Czenczek-Lewandowska E, Lewandowski B, Leszczak J, Podgórska-Bednarz J, Baran J. Occupational activity in patients 10 years after hip replacement surgery. Ortop Traumatol Rehabil. 2016;18:327-36.

62. Nevitt MC, Epstein WV, Masem M, Murray WR. Work disability before and after total hip arthroplasty assessment of effectiveness in reducing disability. Arthritis Rheum. 1984;27:410-21.

63. Ollivier M, Frey S, Parratte S, Flecher X, Argenson JN. Preoperative function, motivation and duration of symptoms predict sporting participation after total hip replacement. Bone Jt J. 2014;96 B:1041-6.

64. Healy WL, Iorio R, Lemos MJ. Athletic activity after joint replacement. Am J Sports Med. 2001;29:377-88.

65. Swanson EA, Schmalzried TP, Dorey FJ. Activity recommendations after total hip and knee arthroplasty: a survey of the American Association for Hip and Knee Surgeons. J Arthroplasty. 2009;24:120-6.

66. Bradley BM, Moul SJ, Doyle FJ, Wilson MJ. Return to sporting activity after total hip arthroplasty - a survey of members of the British Hip Society. J Arthroplasty. 2017;32:898-902.

67. Ollivier M, Frey S, Parratte S, Flecher X, Argenson JN. Does impact sport activity influence total hip arthroplasty durability? Clin Orthop Relat Res. 2012;470:3060-6.

68. Krismer M. Sports activities after total joint arthroplasty. EFORT Open Rev. 2017;2(5):189-94.

69. Kuijer PPFM, De Beer MJPM, Houdijk JHP, Frings-Dresen MHW. Beneficial and limiting factors affecting return to work after total knee and hip arthroplasty: a systematic review. J Occup Rehabil. 2009;19:375-81.

70. Malviya A, Wilson G, Kleim B, Kurtz SM, Deehan D. Factors influencing return to work after hip and knee replacement. Occup Med. 2014;64:402-9.

71. Pahlplatz TMJ, Schafroth MU, Kuijer PPFM. Patient-related and work-related factors play an important role in return to work after total knee arthroplasty: a systematic review. J ISAKOS. 2017;2:127-32.

72. Hoorntje A, Leichtenberg CS, Koenraadt KLM, van Geenen RCI, Kerkhoffs GMMJ, Nelissen RGHH, et al. Not physical activity, but patient beliefs and expectations are associated with return to work after total knee arthroplasty. J Arthroplasty. 2018;33(4):1094-100.

73. Dorey FJ, Amstutz HC. The need to account for patient activity when evaluating the results of total hip arthroplasty with survivorship analysis. J Bone Jt Surg Am. 2002;84-A:709-10. 